\title{
Effectiveness of Respiratory Muscle Training for Pulmonary Function and Walking Ability in Patients with Stroke: A Systematic Review with Meta-Analysis
}

\author{
Diana P. Pozuelo-Carrascosa 1,2,3 $\mathbb{D}$, Juan Manuel Carmona-Torres ${ }^{1,2,4}$ (D), \\ José Alberto Laredo-Aguilera 2,4,5,*iD, Pedro Ángel Latorre-Román ${ }^{6}$, \\ Juan Antonio Párraga-Montilla ${ }^{6}(\mathbb{D})$ and Ana Isabel Cobo-Cuenca ${ }^{1,2,4}(\mathbb{D}$ \\ 1 Faculty of Physiotherapy and Nursing of Toledo, University of Castilla-La Mancha, 45005 Toledo, Spain; \\ dianap.pozuelo@uclm.es (D.P.P.-C.); juanmanuel.carmona@uclm.es (J.M.C.-T.); \\ anaisabel.cobo@uclm.es (A.I.C.-C.) \\ 2 Multidisciplinary Research Group in Care (IMCU), University of Castilla-La Mancha, 45005 Toledo, Spain \\ 3 Social and Health Care Research Center (CESS), University of Castilla-La Mancha, 16071 Cuenca, Spain \\ 4 Maimónides Biomedical Research Institute of Córdoba (IMIBIC), 14004 Córdoba, Spain \\ 5 Faculty of Health Sciences, University of Castilla-La Mancha, Talavera de la Reina, 45600 Toledo, Spain \\ 6 Department of Didactics of Music, Plastic and Corporal Expression, University of Jaén, 23071 Jaén, Spain; \\ platorre@ujaen.es (P.Á.L.-R.); jparraga@ujaen.es (J.A.P.-M.) \\ * Correspondence: josealberto.laredo@uclm.es
}

Received: 19 June 2020; Accepted: 22 July 2020; Published: 24 July 2020

\begin{abstract}
Background: Neurological dysfunction due to stroke affects not only the extremities and trunk muscles but also the respiratory muscles. Aim: to synthesise the evidence available about the effectiveness of respiratory muscle training (RMT) to improve respiratory function parameters and functional capacity in poststroke patients. Methods: a systematic electronic search was performed in the MEDLINE, EMBASE, SPORTDiscus, PEDro and Web of Science databases, from inception to May 2020. Study selection and data extraction: randomised controlled trials (RCTs) that examined the effects of RMT versus non-RMT or sham RMT in poststroke patients. We extracted data about respiratory function, respiratory muscle strength and functional capacity (walking ability, dyspnea, balance, activities of daily life), characteristics of studies and features of RMT interventions (a type of RMT exercise, frequency, intensity and duration). Two reviewers performed study selection and data extraction independently. Results: nineteen RCTs met the study criteria. RMT improved the first second forced expiratory volume (FEV1), forced vital capacity (FVC), peak expiratory flow (PEF), maximal expiratory pressure (MEP), maximal inspiratory pressure (MIP) and walking ability (6 min walking test), but not Barthel index, Berg balance scale, and dyspnea. Conclusions: RMT interventions are effective to improve respiratory function and walking ability in poststroke patients.
\end{abstract}

Keywords: stroke; respiratory muscle training; meta-analysis; walking ability; pulmonary function

\section{Introduction}

Stroke is a loss of focal neurological function due to infarction or haemorrhage in an essential part of the brain [1]. This neurological damage affects not only peripheral but also respiratory muscles, causing respiratory weakness, changes in the respiratory pattern and decreases in respiratory volumes and flows [2,3]. These breathing alterations are related to a decrease in physical activity and therefore, to a reduction in the ability to carry out the activities of daily life [4]. 
Respiratory muscles respond to training similarly to any other skeletal muscle, so just as the upper and lower limb muscles are trained in stroke patients, the respiratory muscles must be trained. In this regard, respiratory muscle training (RMT) consists of repetitive breathing exercises with hand-held respiratory trainer devices to provide pressure threshold or flow-dependent resistance against inhalation (inspiratory muscle training (IMT)) and/or exhalation (expiratory muscle training (EMT)) $[5,6]$ to stimulate this musculature and to produce changes in the muscles' structure.

Previous systematic reviews and meta-analyses have synthesised the available evidence about the effectiveness of RMT exercises on improving respiratory function [3,7-10], even exercise tolerance in poststroke patients; however, some weaknesses of these reviews were that they did not only include randomised controlled trials (RCTs) [10], and that they included a limited number of studies [3,8]. Moreover, since the last systematic review and meta-analysis [7], many studies aiming at analysing the effect of RMT on respiratory function and functional capacity have been published.

For these reasons, this systematic review and meta-analysis aimed to synthetize the most novel evidence about the effectiveness of RMT to improve respiratory function, respiratory muscle strength and functional capacity in poststroke patients.

\section{Materials and Methods}

This meta-analysis was performed in accordance with the Preferred Reporting Items for Systematic Reviews and Meta-Analyses (PRISMA) [11] and following the recommendations of the Cochrane Handbook for Systematic Reviews of Interventions [12]. Additionally, this systematic review and meta-analysis was registered through PROSPERO (awaiting register number; ID: 182082).

\subsection{Search Strategy}

Two researchers (DPP-C and AIC-C) independently searched MEDLINE (via Pubmed), EMBASE (via Scopus), SPORTDiscus, PEDro and Web of Science databases from inception to May 2020; disagreements were solved by consensus or involving a third researcher (JAL-A). The search strategy used was as follow: ("inspiratory muscle training" OR "IMT" OR "expiratory muscle training" OR "respiratory therapy" OR "chest physiotherapy" OR "respiratory exercise" OR "breathing exercises" AND ("stroke" OR "acute stroke" OR "cerebral stroke") AND ("pulmonary function" OR "respiratory function" OR "exercise tolerance" OR "walking ability" OR "gait ability") AND (random* control* trials). The literature search was complemented by scanning the reference list of the included articles, and the list of references of previous systematic reviews was reviewed for additional relevant studies.

\subsection{Study Selection}

Reviewers were not blinded to authors, journals, or institutions. Included articles were RCTs studies that analysed the effectiveness of RMT (IMT and/or EMT) on improving pulmonary function, respiratory muscle strength and functional capacity parameters in patients with stroke.

The inclusion criteria were: (1) Patients: adults with stroke (haemorrhagic or ischaemic); (2) Intervention: IMT, EMT or both; (3) Control: no respiratory training or sham respiratory muscle training (without any resistance); (4) Outcomes: variables of pulmonary function such as the first second forced expiratory volume (FEV1), forced vital capacity (FVC), and peak expiratory flow (PEF); parameters of respiratory muscle weakness: maximal expiratory pressure (MEP) and maximal inspiratory pressure (MIP); Functional capacity including 6 min walking test (6-MWT), Barthel index (MBI), Berg balance scale (BBS), and dyspnea (Borg scale); (5) type of studies: RCTs.

Exclusion criteria were: (1) studies not written in English or Spanish; (2) studies not reporting the outcome variables; (3) non-RCTs; (4) insufficient data.

\subsection{Data Extraction}

For data extraction, two authors (DPP-C and AIC-C), independently, used a standardised data collection form. From each selected study, the following data were collected: (1) first author's name and 
year of publication; (2) country; (3) period of data collection; (4) characteristics of study sample: sample size, age mean, gender; (5) data concerning inclusion/exclusion criteria; (6) main study outcomes. We emailed the corresponding author requesting the data when some information was lacking.

\subsection{Risk of Bias Assessment}

While in the Prospero register we stated that the intent to use the Cochrane Collaboration tool to assess the risk of bias (RoB2) [13], we observed that all previous systematic reviews and meta-analysis on this topic had used the PEDro Scale to assess the quality of included studies. Thus, with the aim of increase the homogeneity and the comparability, we decided to use this scale instead of RoB2.

PEDro Scale is a useful tool for assessing the quality of physical therapy trials [14]. This scale has 11 items designed for rating the methodological quality of RCTs assessing the internal validity (e.g., random and concealed allocation, baseline similarity, blinding, and intention-to-treat analysis) and statistical reporting. The total PEDro score ranges from 0 to 10 points (the first item, eligibility criteria, is not included in the total score); higher score means better study's quality [15].

Data extraction and risk of bias assessment were independently completed by two reviewers (DPP-C and AIC-C) if appeared inconsistencies were solved by consensus or involving a third researcher (JAL-A).

\subsection{Statistical Analysis}

For each of the main outcomes (FEV1, MIP, MEP, PEF, FVC, 6-MWT, dyspnea, MBI and BBS), a separate pooled estimate of effect size (ES) and their respective $95 \%$ confidence intervals ( $95 \% \mathrm{CI}$ ) was calculated. The ES parameters from preintervention to postintervention between groups (exercise intervention vs control) [16] in each study were calculated assuming a correlation coefficient of 0.5 and using a random-effects model based on the DerSimonian and Laird method [17]. Statistical heterogeneity was analysed by the I2 statistic. Heterogeneity was considered as not important (I2: $0 \%$ to $40 \%$ ), moderate (I2: $30 \%$ to $60 \%$ ), substantial (I2: $50 \%$ to $90 \%$ ) or considerable (I2: $75 \%$ to $100 \%$ ); the corresponding $p$-values were considered [12]. When a study reported results separately for IMT and EMT, we calculated a combined estimate.

According to the Cochrane Handbook recommendations, when mean and standard deviation (SD) were lacking, and available statistics were median and interquartile range (IQR), the IQR was divided by 1.35 to transform these estimates on CI [18]. Finally, when studies were scaled inversely (i.e., lower values indicated worse outcomes), the mean in each group was multiplied by -1 .

Subgroup analyses were conducted when it was possible due to the number of studies, to assess whether the intervention of RMT (IMT/EMT) or only IMT allowed better results on outcome variables. Sensitivity analyses were performed by removing studies one by one to assess the robustness of the summary estimates and to detect whether any study accounted for a large proportion of heterogeneity among RMT and ES pooled estimations. Meaningful results of the sensitivity analyses were considered when the resulting estimates were modified beyond the CIs of the original summary estimate.

Finally, Egger regression asymmetry method and visual inspection of funnel plots were used to assess publication bias. Statistical analyses were performed using Stata Statistical software, version 16.0 (Stata, College Station, TX, USA).

\section{Results}

The electronic search retrieved 826 studies, and we identified two new studies through manual search. After removing duplicated studies, the title and abstract of 800 studies were revised. Of these, 754 were excluded by irrelevancy and 46 were full-text revised. Finally, nineteen studies aimed to assess the effectiveness of RMT on pulmonary function and functional capacity variables in patients with stroke were included in the meta-analysis [19-37]. Figure 1 shows the flow of study selection. The reasons for the full-text studies excluded are summarised in Supplementary File S1. 


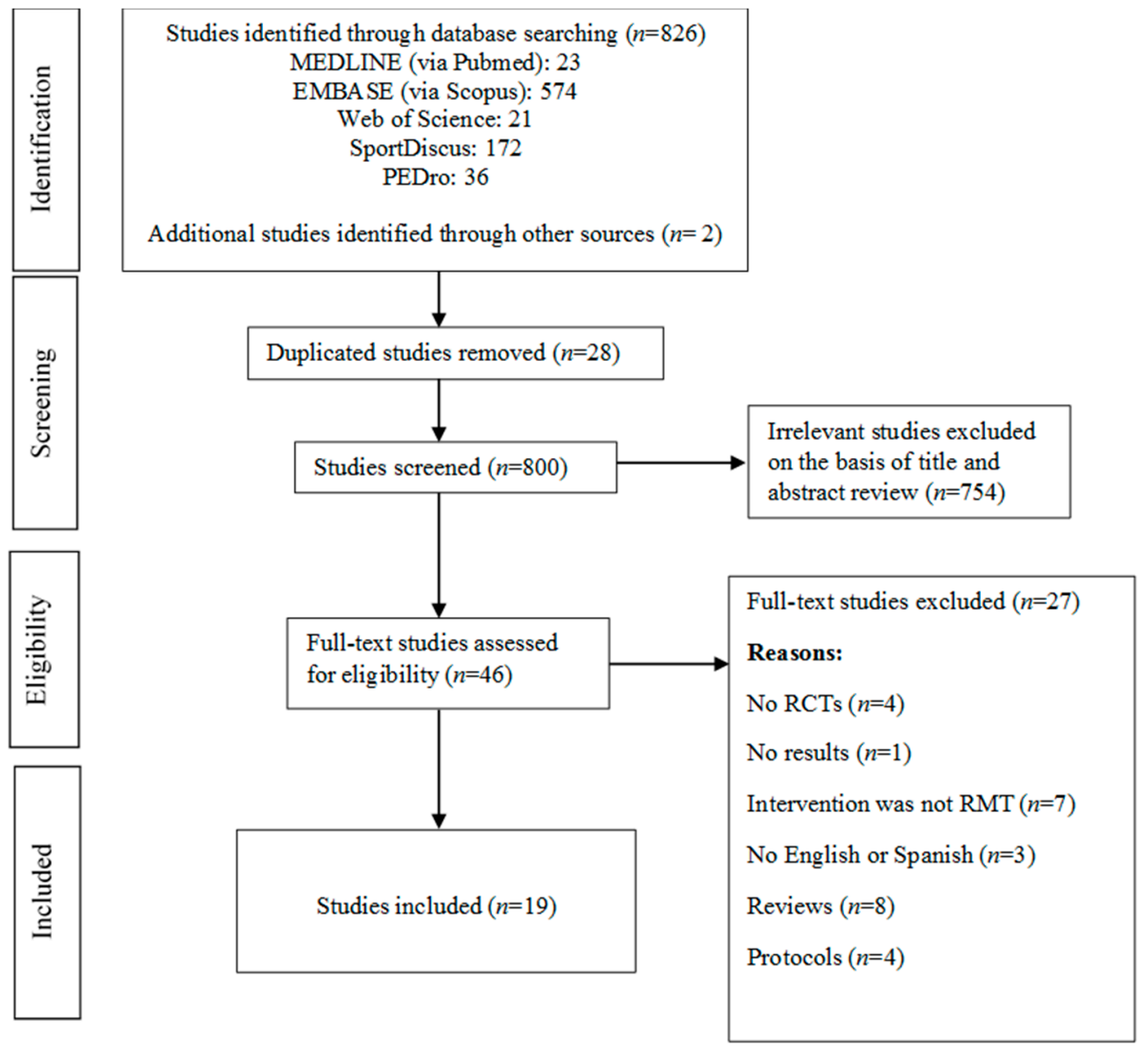

Figure 1. Literature search: Preferred Reporting Items for Systematic Reviews and Meta-Analyses (PRISMA) consort diagram. RMT, Respiratory muscle training; RCTs, randomized controlled trials.

Table 1 depicts the characteristics of the included studies and the participants. Studies were conducted in Korea [20,22-27,33,34,36], Turkey, Spain [28,30], Brazil [21,35], Germany [32], United Kingdom [31] and Taiwan [19,29].

The nineteen studies included involved 643 patients with stroke, with the sample size varying between 12 and 82 participants. The mean age of participants was 61.3 years.

All included studies performed RMT; nevertheless, ten studies carried out IMT and EMT [19-21, $23,28,30-33,36]$, and nine studies only performed IMT [22,24-27,29,34,35,37]. Regarding the threshold devices used in the RMT interventions, they were very varied: Respironics, Respifit-S, Orygen Dual Valve, SpiroTiger, Threshold, PowerBreath, tri-ball incentive spirometer and Dofin Breathing (Table 1).

The interventions were very diverse; the number of sets per session varied between two and ten sets; the repetitions in each set ranged between five and 30. Other studies listed time rather than the number of repetitions; the load of RMT in the majority of studies was at $30 \%$ of MIP/MEP at the beginning, increasing with the intervention. However, some studies used higher loads at $40 \%$ or $50 \%$ at the beginning of the intervention.

The length of the interventions ranged between three and ten weeks. In the control group, most studies conducted the conventional stroke rehabilitation program, however, four out of twenty studies performed sham respiratory training without resistance and/or progression $[21,30,31,35]$. 
Table 1. Characteristics of studies included.

\begin{tabular}{|c|c|c|c|c|c|c|}
\hline Study & Country & $\begin{array}{l}\text { Participants/ } \\
\text { Mean Age }\end{array}$ & Inclusion/Exclusion Criteria & Intervention & Control & Study Outcomes \\
\hline $\begin{array}{l}\text { Liaw et al., } \\
2020 \text { [19] }\end{array}$ & Taiwan & $\begin{array}{l}n=31 \\
\text { GI: } 15 \\
\text { GC: } 16 \\
38.7 \% \text { men } \\
62.8 \text { years }\end{array}$ & $\begin{array}{l}\text { Inclusion: } \\
\text { Aged } 35 \text { to } 80 \text { years with unilateral stroke }>6 \\
\text { months, respiratory muscle weakness, } \\
\text { dysphagia, or dysarthria. } \\
\text { Exclusion: } \\
\text { Increased intracranial pressure, uncontrolled } \\
\text { hypertension, decompensated heart failure, } \\
\text { unstable angina, recent myocardial infarction, } \\
\text { complicated arrhythmias, pneumothorax, } \\
\text { bullae/blebs in the preceding } 3 \text { months, severe } \\
\text { cognitive function or infection, recurrent } \\
\text { stroke, brain stem stroke, and aphasia. }\end{array}$ & $\begin{array}{l}\text { IMT load was } 30 \%-60 \% \text { of } \\
\text { MIP ( } 6 \text { sets of } 5 \text { repetitions). } \\
\text { EMT load was } 15 \%-75 \% \text { of } \\
\text { MEP ( } 5 \text { sets of } 5 \text { breaths). } \\
\text { Session: IMT and EMT, } 1 \text { to } 2 \\
\text { times per day, } 5 \text { days a week } \\
\text { for } 6 \text { weeks, } 1 \text { to } 2 \text { min between } \\
\text { each set. } \\
\text { Device: Dofin Breathing Trainer } \\
\text { (DT } 11 \text { or DT } 14 \text { ). } \\
\text { RHB program as CG. }\end{array}$ & $\begin{array}{l}\text { RHB program: postural } \\
\text { training, breathing control, } \\
\text { improving cough } \\
\text { technique, checking chest } \\
\text { wall mobility, fatigue } \\
\text { management, orofacial } \\
\text { exercises, thermal tactile } \\
\text { stimulation, Mendelsohn } \\
\text { maneuvering, effort } \\
\text { swallowing, or } \\
\text { supra-glottic maneuver. }\end{array}$ & $\begin{array}{l}\text {-FEV1 } \\
\text {-MEP } \\
\text {-MIP } \\
\text {-MBI } \\
\text {-Dyspnea } \\
\text {-FVC }\end{array}$ \\
\hline $\begin{array}{l}\text { Lee et al., } \\
2019 \text { [20] }\end{array}$ & $\begin{array}{l}\text { Seoul, } \\
\text { Korea }\end{array}$ & $\begin{array}{l}n=25 \\
\text { GI: } 13 \\
\text { GC: } 12 \\
48 \% \text { men } \\
59.1 \text { years }\end{array}$ & $\begin{array}{l}\text { Inclusion: } \\
\text { Stroke over } 6 \text { months, MMSE }>24 \text {, no facial } \\
\text { palsy and receptive aphasia, and no prior } \\
\text { thoracic or abdominal surgery. } \\
\text { Exclusion: } \\
\text { Medications for neuromuscular control or that } \\
\text { provoke drowsiness, significant disability } \\
\text { prior to stroke as evidenced by a score }>3 \text { on } \\
\text { MRS, restrictive lung disease, TIS }<10 \text {, and } \\
\text { musculoskeletal problems in the pelvis or } \\
\text { spine. }\end{array}$ & $\begin{array}{l}\text { IMT/EMT at } 30 \% \text { of the } \\
\text { resistance intensity on the first } \\
\text { day of the week. } \\
\text { Session: } 10-15 \text { times, } 5 \text { set for } 20 \\
\text { min in a session and resting } \\
\text { time of } 30-60 \text { s between each } \\
\text { set. } \\
\text { Trunk stabilization exercises: } \\
40 \text { min, } 3 \text { times a week for } 6 \\
\text { weeks. } \\
\text { Device: Threshold PEP; } \\
\text { Threshold IMT-Respironics. } \\
\text { RHB program as CG. }\end{array}$ & $\begin{array}{l}\text { Conventional physical and } \\
\text { occupational therapy } \\
\text { conducted for } 30 \text { min, } 2 \\
\text { times a day, and } 6 \text { times per } \\
\text { week. } \\
\text { Trunk stabilization } \\
\text { exercises: } 40 \text { min, } 3 \text { times a } \\
\text { week for } 6 \text { weeks. }\end{array}$ & $\begin{array}{l}\text {-FEV1 } \\
-\mathrm{MEP} \\
-\mathrm{MIP} \\
-\mathrm{PEF}\end{array}$ \\
\hline $\begin{array}{l}\text { Menezes et } \\
\text { al., } 2019 \text { [21] }\end{array}$ & Brazil & $\begin{array}{l}n=38 \\
\text { GI: } 19 \\
\text { GC: } 19 \\
50 \% \text { men } \\
63.5 \text { years }\end{array}$ & $\begin{array}{l}\text { Inclusion: } \\
\text { Adults }>20 \text { years, stroke }>3 \text { months and }<5 \\
\text { years, } \mathrm{MIP}<80 \mathrm{~cm} \mathrm{H}_{2} \mathrm{O} \text { or } \mathrm{MEP}<90 \mathrm{~cm} \mathrm{H}_{2} \mathrm{O} \\
\text { (respiratory muscle weakness), and not } \\
\text { undertook any respiratory training. } \\
\text { Exclusion: } \\
\text { Patients with cognitive deficits, facial palsy, } \\
\text { and/or any conditions. }\end{array}$ & $\begin{array}{l}\text { Home based IMT/EMT. } \\
\text { Session: } 40 \text { min/day (two } 20 \\
\text { min sessions), } 7 \text { times/ week, } \\
\text { over } 8 \text { weeks. } \\
\text { Each } 20 \text {-min session: } 4 \text { min sets } \\
\text { of training, followed by } 1 \text { min } \\
\text { rest. } \\
\text { Load: } 50 \% \text { MIP/MEP } \\
\text { Device: Orygen-dual valve. }\end{array}$ & $\begin{array}{l}\text { Sham respiratory training } \\
\text { without any resistance and } \\
\text { progression. }\end{array}$ & $\begin{array}{l}\text {-MIP } \\
\text {-MEP } \\
\text {-Dyspnea } \\
\text {-6-MWT }\end{array}$ \\
\hline
\end{tabular}


Table 1. Cont.

\begin{tabular}{|c|c|c|c|c|c|c|}
\hline Study & Country & $\begin{array}{l}\text { Participants/ } \\
\text { Mean Age }\end{array}$ & Inclusion/Exclusion Criteria & Intervention & Control & Study Outcomes \\
\hline $\begin{array}{l}\text { Cho et al., } \\
2018 \text { [22] }\end{array}$ & Korea & $\begin{array}{l}n=25 \\
\text { GI: } 12 \\
\text { GC: } 13 \\
52 \% \text { men } \\
49.5 \text { years }\end{array}$ & $\begin{array}{l}\text { Inclusion: } \\
\text { Adults }>20 \text { years, stroke }>3 \text { months, MIP }< \\
70 \% \text { of those predicted when adjusted for age } \\
\text { and sex, had and were able to give informed } \\
\text { consent and follow study procedures. } \\
\text { Exclusion: } \\
\text { Patients with facial palsy, myocardial } \\
\text { infarction or acute heart failure within } 3 \\
\text { months, pulmonary disease, neurological } \\
\text { conditions or medications that interfere with } \\
\text { neuromuscular control. }\end{array}$ & $\begin{array}{l}\text { Hospital IMT. } \\
\text { Session: } 3 \text { sets of } 30 \text { breaths, } \\
\text { with a } 1 \text {-min rest in between } \\
\text { the sets. } 5 \text { days a week, for } 6 \\
\text { weeks. } \\
\text { Load: } 30 \% \text { MIP readjusted } \\
\text { weekly. } \\
\text { Device: PowerBreath K5. } \\
\text { RHB program as CG. }\end{array}$ & $\begin{array}{l}\text { RHB program: muscle } \\
\text { strengthening exercises, } \\
\text { Bobath therapy, gait } \\
\text { training, and stair climbing } \\
\text { training for } 60 \text { min/day, } 5 \\
\text { days per week, for } 6 \text { weeks. }\end{array}$ & $\begin{array}{l}\text {-MIP } \\
\text {-Fatigue } \\
\text {-6-MWT }\end{array}$ \\
\hline $\begin{array}{l}\text { Yoo et al., } \\
2018 \text { [23] }\end{array}$ & $\begin{array}{l}\text { Seoul, } \\
\text { Korea }\end{array}$ & $\begin{array}{l}n=40 \\
\text { GI: } 20 \\
\text { GC: } 20 \\
65 \% \text { men } \\
61 \text { years }\end{array}$ & $\begin{array}{l}\text { Inclusion: } \\
\text { Adults > } 18 \text { years; first episode of stroke } \\
\text { within } 3 \text { months; moderate to severe stroke } \\
\text { impairment; understand instructions and } \\
\text { follow the study program. } \\
\text { Exclusion: } \\
\text { Persistent cardiopulmonary disease, } \\
\text { coexisting brain disorders, uncontrolled } \\
\text { hypertension, severe facial palsy or other } \\
\text { oropharyngeal structural abnormality, severe } \\
\text { oral apraxia, and tracheostomy. }\end{array}$ & $\begin{array}{l}\text { Bedside IMT/EMT. } \\
\text { Session: } 10 \text { min of breath } \\
\text { stacking exercise }+10 \text { min IMT } \\
+10 \text { min EMT; with a } 5 \text {-min } \\
\text { interval before each exercise. } \\
\text { Twice/day for } 7 \text { days/week for } \\
\text { a 3-wk period. } \\
\text { Device: A flow-oriented } \\
\text { incentive spirometer } \\
\text { (Hyupsung) for IMT; Acapella } \\
\text { vibratory PEP for EMT. } \\
\text { RHB program as CG. }\end{array}$ & $\begin{array}{l}\text { Conventional stroke RHB } \\
\text { program: motion exercises, } \\
\text { muscle strengthening, gait } \\
\text { training, fine motor } \\
\text { exercises, and activity of } \\
\text { daily living training. It was } \\
\text { performed for } 30 \text { min, } \\
\text { twice a day } 5 \text { days a week, } \\
\text { for } 3 \text { weeks. }\end{array}$ & $\begin{array}{l}-\mathrm{FVC} \\
-\mathrm{FEV1} \\
-\mathrm{PEF} \\
-\mathrm{MBI} \\
-\mathrm{BBS}\end{array}$ \\
\hline $\begin{array}{l}\text { Lee et al., } \\
2018 \text { [24] }\end{array}$ & $\begin{array}{l}\text { Jeonju, } \\
\text { Korea }\end{array}$ & $\begin{array}{l}n=20 \\
\text { GI: } 10 \\
\text { GC: } 10 \\
50 \% \text { men } \\
60 \text { years }\end{array}$ & $\begin{array}{l}\text { Inclusion: } \\
\text { Patients with hemiplegia due to stroke, } 50-70 \\
\text { years, diagnosed 1-2 year previously, could } \\
\text { perform } 10-\mathrm{m} \text { walking independently and } \\
\text { walk within } 5-60 \text { sec, ability to understand } \\
\text { and follow the indication of the researcher. } \\
\text { Exclusion: NA }\end{array}$ & $\begin{array}{l}\text { Load of IMT: } 50 \% \text { of MIP } \\
\text { increasing repetitions each } \\
\text { week. } \\
\text { Session: } 20 \text { min of IMT and } 20 \\
\text { min of bracing exercise for } 6 \\
\text { weeks and } 5 \text { days per week. } \\
\text { Bracing exercise holding the } \\
\text { pressometer at } 40 \text { mmHg. } \\
\text { Device: POWERbreath for IMT. } \\
\text { RHB program as CG. }\end{array}$ & $\begin{array}{l}\text { Traditional exercise to } \\
\text { enhance trunk control } \\
\text { ability and included a } \\
\text { stretching exercise for trunk } \\
\text { flexibility, for } 6 \text { weeks, } 5 \\
\text { days per week, once for } 40 \\
\text { min. }\end{array}$ & $\begin{array}{l}\text {-6-MWT } \\
\text {-BBS }\end{array}$ \\
\hline
\end{tabular}


Table 1. Cont.

\begin{tabular}{|c|c|c|c|c|c|c|}
\hline Study & Country & $\begin{array}{l}\text { Participants/ } \\
\text { Mean Age }\end{array}$ & Inclusion/Exclusion Criteria & Intervention & Control & Study Outcomes \\
\hline $\begin{array}{l}\text { KM Jung, } \\
2017 \text { [25] }\end{array}$ & Korea & $\begin{array}{l}n=12 \\
\text { GI: } 6 \\
\text { GC: } 6 \\
41.6 \% \text { men } \\
61.7 \text { years }\end{array}$ & $\begin{array}{l}\text { Inclusion: } \\
\text { Hemiparesis due to stroke, event occurring }<6 \\
\text { months previously, ability to use a cycle } \\
\text { ergometer, no restriction in lung function and } \\
\text { no neurologic, orthopedic, or unstable cardiac } \\
\text { condition, and ability to walk } 100 \mathrm{~m} \text {. } \\
\text { Exclusion: } \\
\text { Comorbidities or disabilities that would } \\
\text { preclude study, and any uncontrolled health } \\
\text { condition for which exercise is } \\
\text { contraindicated. }\end{array}$ & $\begin{array}{l}\text { Session: IMT } 6 \text { sets of } 5 \text { min } \\
\text { each for } 30 \text { min a day, } 5 \text { times a } \\
\text { week, for } 4 \text { weeks. } \\
\text { Load: IMT at } 30 \% \text { MIP. } \\
\text { Device: Threshold IMT. } \\
\text { Moreover, traditional physical } \\
\text { therapy and occupational } \\
\text { therapy. }\end{array}$ & $\begin{array}{l}\text { A self-selective intensity } \\
\text { exercise with an ergonomic } \\
\text { cycle for } 30 \text { min a day, five } \\
\text { times a week, for four } \\
\text { weeks. }\end{array}$ & $\begin{array}{l}-\mathrm{FVC} \\
\text {-FEV1 } \\
-6-\mathrm{MWT}\end{array}$ \\
\hline $\begin{array}{l}\text { NJ Jung, } \\
2017 \text { [26] }\end{array}$ & $\begin{array}{l}\text { Daegu, } \\
\text { Korea }\end{array}$ & $\begin{array}{l}n=20 \\
\text { GI: } 10 \\
\text { GC: } 10 \\
\text { NA }\end{array}$ & $\begin{array}{l}\text { Inclusion: } \\
\text { Stroke } 6 \text { months or longer; had no visual field } \\
\text { defect and auditory sense; Scored at least } 24 \\
\text { on the K-MMSE; independent sitting and gait; } \\
\text { had no pulmonary embolus; no orthopedic } \\
\text { problem, or unstable cardiac condition; had } \\
\text { not undergone chest or abdominal surgery. } \\
\text { Exclusion: NA }\end{array}$ & $\begin{array}{l}\text { Session: } 2 \text { sets of } 10 \text { times IMT, } \\
\text { taking a break of } 10 \text { s between } \\
\text { each set. } 5 \text { times a week, for } 6 \\
\text { weeks. } \\
\text { Load of IMT: } 80 \% \text { of MIP } \\
\text { Device: Respifit-S. } \\
\text { Neuro Developmental } \\
\text { Treatment as CG. }\end{array}$ & $\begin{array}{l}\text { Neuro developmental } \\
\text { treatment physical therapy } \\
\text { for } 30 \text { min per time, } 3 \text { times } \\
\text { a week, for } 6 \text { weeks. }\end{array}$ & -6-MWT \\
\hline $\begin{array}{l}\text { Guillen-Sola } \\
\text { et al., } 2017 \\
\text { [28] }\end{array}$ & Spain & $\begin{array}{l}n=50 \\
\text { GI1: } 16 \\
\text { GI2: } 16 \\
\text { GC: } 18 \\
51.3 \% \text { men } \\
69.0 \text { years }\end{array}$ & $\begin{array}{l}\text { Inclusion: } \\
\text { Subacute ischemic stroke within } 1 \text { to } 3 \text { weeks } \\
\text { of inclusion and dysphagia. } \\
\text { Exclusion: } \\
\text { History of previous neurological diseases } \\
\text { and/or cognitive impairment }\end{array}$ & $\begin{array}{l}\text { Session: IMT/EMT, } 5 \text { sets of } 10 \\
\text { breaths followed by } 1 \text { min of } \\
\text { unloaded recovery breathing } \\
\text { off the device. Twice a day, } 5 \\
\text { days per week for } 3 \text { weeks, } \\
\text { with the assistance of a } \\
\text { therapist. } \\
\text { Load: } 30 \% \text { of MIP/MEP and } \\
\text { increased weekly at intervals of } \\
10 \mathrm{~cm} \mathrm{H}_{2} \mathrm{O} \text {. } \\
\text { Device: Orygen dual valve. } \\
\text { RHB program as CG. }\end{array}$ & $\begin{array}{l}\text { RHB program: } \\
\text { Physical, occupational and } \\
\text { speech therapy targeting } \\
\text { specific impairments in } \\
\text { mobility, activities of daily } \\
\text { living, swallowing and } \\
\text { communication skills. }\end{array}$ & $\begin{array}{l}\text {-MIP } \\
\text {-MEP }\end{array}$ \\
\hline
\end{tabular}


Table 1. Cont.

\begin{tabular}{|c|c|c|c|c|c|c|}
\hline Study & Country & $\begin{array}{l}\text { Participants/ } \\
\text { Mean Age }\end{array}$ & Inclusion/Exclusion Criteria & Intervention & Control & Study Outcomes \\
\hline $\begin{array}{l}\text { Oh et al., } \\
2016 \text { [27] }\end{array}$ & Korea & $\begin{array}{l}n=23 \\
\text { GI: } 11 \\
\text { GC: } 12 \\
56.5 \% \text { men } \\
70.6 \text { years }\end{array}$ & $\begin{array}{l}\text { Inclusion: } \\
\text { Unilateral stroke occurred } 6 \text { months prior to } \\
\text { the study the ability to perform breathing } \\
\text { training for } 30 \text { min or longer; no sight } \\
\text { impairment; modified Ashworth scale (MAS) } \\
\text { score for upper and low extremities }<2 \text {; and } \\
\text { MMSE-K score }>24 \text {. } \\
\text { Exclusion: NA }\end{array}$ & $\begin{array}{l}\text { Session: } 15 \text { times per set, } 10 \text { sets. } \\
\text { Warm-up and cool-down } \\
\text { exercises twice in each set, with } \\
\text { a rest time of } 60 \mathrm{s.} .20 \text { min per } \\
\text { day, } 3 \text { times per week for } 6 \\
\text { weeks. } \\
\text { Load: IMT resistance } 30 \% \text { MIP. } \\
\text { Device: } n / \text { A. } \\
\text { RHB program as CG. }\end{array}$ & $\begin{array}{l}\text { RHB program: } \\
\text { 20-min session, } 3 \\
\text { times/week, for } 6 \text { weeks. } \\
\text { General breathing exercises, } \\
\text { abdominal strengthening } \\
\text { exercises, and general } \\
\text { physical therapy. }\end{array}$ & $\begin{array}{l}-\mathrm{FVC} \\
-\mathrm{FEV1} \\
-\mathrm{PEF} \\
-\mathrm{BBS}\end{array}$ \\
\hline $\begin{array}{l}\text { Chen et al., } \\
2016 \text { [29] }\end{array}$ & Taiwan & $\begin{array}{l}n=21 \\
\text { GI: } 11 \\
\text { GC: } 10 \\
38.1 \% \text { men } \\
65.5 \text { years }\end{array}$ & $\begin{array}{l}\text { Inclusion: } \\
\text { Adults } 20-85 \text { years with congestive heart } \\
\text { failure and stroke. } \\
\text { Exclusion: } \\
\text { MIP } \geq 70 \% \text { predicted MIP, could not tightly } \\
\text { place the lips over a mouthpiece, recent acute } \\
\text { exacerbation of COPD, pneumothorax or } \\
\text { large bullae on chest radiography, long-term } \\
\text { use of oxygen therapy, recent lung surgery } \\
\text { marked osteoporosis, unstable angina, } \\
\text { decompensated CHF, or arrhythmia. }\end{array}$ & $\begin{array}{l}\text { Session: IMT with a load of } 30 \% \\
\text { of MIP (adjusted by } 2 \mathrm{~cm} \\
\mathrm{H}_{2} \mathrm{O} / \text { week), for } 30 \text { min, } 5 \\
\text { times/week, for } 10 \text { weeks. } \\
\text { Device: Respironics. } \\
\text { RHB program as CG. }\end{array}$ & $\begin{array}{l}\text { Conventional stroke RHB } \\
\text { program, } 5 \text { days/week for } \\
10 \text { weeks. }\end{array}$ & $\begin{array}{l}\text {-FVC } \\
\text {-FEV1 } \\
\text {-Dyspnea } \\
\text {-MBI }\end{array}$ \\
\hline $\begin{array}{l}\text { Messaggi- } \\
\text { Sartor et al., } \\
2015 \text { [30] }\end{array}$ & Spain & $\begin{array}{l}n=77 \\
\text { GI: } 39 \\
\text { GC: } 38 \\
57.8 \% \text { men } \\
66.5 \text { years }\end{array}$ & $\begin{array}{l}\text { Inclusion: } \\
\text { Adults, first-ever ischemic stroke, time since } \\
\text { stroke }<3 \text { weeks, hemiparesis in upper or } \\
\text { lower limb, and gave informed consent and } \\
\text { followed study procedures. } \\
\text { Exclusion: } \\
\text { Cardiopulmonary disease, neurologic } \\
\text { condition other than stroke, significant alcohol } \\
\text { abuse, medical treatment with potential effect } \\
\text { on muscle structure and function. }\end{array}$ & $\begin{array}{l}\text { IMT/EMT with workload of } \\
30 \% \text { MIP/MEP. } \\
\text { Session: } 5 \text { sets of } 10 \text { repetitions } \\
\text { followed by } 1-2 \text { min of } \\
\text { unloaded recovery, twice a day, } \\
5 \text { days per week, for } 3 \text { weeks. } \\
15-20 \text { breaths/min } \\
\text { Device: Orygen-Dual valve. }\end{array}$ & $\begin{array}{l}\text { RHB program: physical, } \\
\text { occupational, and speech } \\
\text { therapy sessions ( } 3 \mathrm{~h} \text { per } \\
\text { day, } 5 \text { days a week, } \\
\text { for } 3 \text { weeks). } \\
\text { Sham IEMT without load } \\
\text { and progression. }\end{array}$ & $\begin{array}{l}\text {-MIP } \\
\text {-MEP }\end{array}$ \\
\hline
\end{tabular}


Table 1. Cont.

\begin{tabular}{|c|c|c|c|c|c|c|}
\hline Study & Country & $\begin{array}{l}\text { Participants/ } \\
\text { Mean Age }\end{array}$ & Inclusion/Exclusion Criteria & Intervention & Control & Study Outcomes \\
\hline $\begin{array}{l}\text { Kulnik et al., } \\
2015 \text { [31] }\end{array}$ & $\begin{array}{l}\text { United } \\
\text { Kingdom }\end{array}$ & $\begin{array}{l}n=82 \\
\text { GI1: } 27 \\
\text { GI2:26 } \\
\text { GC: } 25 \\
57.3 \% \text { men } \\
64.4 \text { years }\end{array}$ & $\begin{array}{l}\text { Inclusion: } \\
\text { Adults, < } 2 \text { weeks of stroke, } 5-25 \text { score in } \\
\text { NIHSS, ability to give informed consent and } \\
\text { follow study procedures. } \\
\text { Exclusion: } \\
\text { Blood pressure }>180 / 100 \mathrm{mmHg} \text {, angina, } \\
\text { myocardial infarction, or acute heart failure } \\
\text { within } 3 \text { months, pulmonary disease; and } \\
\text { neurological conditions other than stroke. }\end{array}$ & $\begin{array}{l}\text { EMT (GI1) and IMT (GI2) } \\
\text { Session: } 5 \text { sets of } 10 \text { breaths } \\
\text { each, with 1-min rests between } \\
\text { sets. } \\
\text { Load was } 50 \% \text { of MIP or MEP. } \\
\text { These pressures were } \\
\text { reassessed, and resistance } \\
\text { readjusted weekly. } \\
\text { Device: Respironics }\end{array}$ & $\begin{array}{l}\text { Sham training: without } \\
\text { load and progression. }\end{array}$ & $\begin{array}{l}\text {-MIP } \\
\text {-MEP }\end{array}$ \\
\hline $\begin{array}{l}\text { CY Kim et } \\
\text { al., } 2015 \text { [32] }\end{array}$ & Germany & $\begin{array}{l}n=37 \\
\text { GI1: } 15 \\
\text { GI2: } 13 \\
\text { GC: } 12 \\
45.94 \% \text { men } \\
59.1 \text { years }\end{array}$ & $\begin{array}{l}\text { Inclusion: } \\
\text { Ischemic or hemorrhagic post-stroke } \\
\text { hemiparesis; K-MMSE } \geq 26 \text {. } \\
\text { Exclusion: } \\
\text { Previous musculoskeletal abnormalities, } \\
\text { confusion, neurological disorders, significant } \\
\text { perceptual, cognitive, or communication } \\
\text { impairments, COPD, and asthma. }\end{array}$ & $\begin{array}{l}\text { Session: IMT/EMT } 5 \text { sets }(10 \\
\text { repetitions/set), } 1 \text {-min of rest } \\
\text { after each set. } \\
30 \text { supervised sessions }(5 \times 15 \\
\text { min/week, } 6 \text { weeks). } \\
\text { Device: Tri-ball Incentive } \\
\text { Spirometer. } \\
\text { RHB program as CG. }\end{array}$ & $\begin{array}{l}\text { RHB program: stretching } \\
\text { exercises of the limbs, } \\
\text { therapist-guided } \\
\text { techniques for facilitating } \\
\text { the normal movement } \\
\text { pattern. } 1 \mathrm{~h}, 5 \text { times a week. }\end{array}$ & $\begin{array}{l}\text {-FEV1 } \\
\text {-FVC }\end{array}$ \\
\hline $\begin{array}{l}\text { J Kim et al., } \\
2014 \text { [33] }\end{array}$ & Korea & $\begin{array}{l}n=20 \\
\text { GI: } 10 \\
\text { GC: } 10 \\
54 \text { years }\end{array}$ & $\begin{array}{l}\text { Inclusion: } \\
\text { Hemiparesis due to stroke in previous } 6 \\
\text { months, capable of comprehending } \\
\text { commands and walking for at least } 6 \text { min } \\
\text { with/without assistive device, had no } \\
\text { previous cardiovascular or respiratory } \\
\text { problems, no medications that would } \\
\text { influence the metabolic or cardiorespiratory } \\
\text { responses to exercise, no previous exercise } \\
\text { training ventilator muscles and no bone } \\
\text { deformities of the chest or spine. } \\
\text { Exclusion: NA }\end{array}$ & $\begin{array}{l}\text { Session: IMT/EMT during } 20 \\
\text { min. Individually loaded and } \\
\text { set to the breathing capacity of } \\
\text { each patient. } \\
\text { Device: Respifit-S. }\end{array}$ & $\begin{array}{l}\text { Conventional exercise } \\
\text { treatments } \\
\text { for } 30 \text { min (including joint } \\
\text { mobility, eccentric } \\
\text { contraction, muscle } \\
\text { strengthening, and walking } \\
\text { exercise) followed by a } \\
\text { 10-min rest. Full body } \\
\text { workout machine for } 20 \\
\text { min. } 3 \text { times/week, for } 4 \\
\text { weeks. }\end{array}$ & $\begin{array}{l}\text {-FEV1 } \\
\text {-PEF } \\
\text {-6-MWT } \\
\text {-Dyspnea }\end{array}$ \\
\hline
\end{tabular}


Table 1. Cont.

\begin{tabular}{|c|c|c|c|c|c|c|}
\hline Study & Country & $\begin{array}{l}\text { Participants/ } \\
\text { Mean Age }\end{array}$ & Inclusion/Exclusion Criteria & Intervention & Control & Study Outcomes \\
\hline $\begin{array}{l}\text { Jung and } \\
\text { Kim, } 2013 \\
{[34]}\end{array}$ & Korea & $\begin{array}{l}n=29 \\
\text { GI: } 15 \\
\text { GC: } 14 \\
58.6 \% \text { men } \\
59 \text { years }\end{array}$ & $\begin{array}{l}\text { Inclusion: } \\
\text { Hemiplegia secondary to stroke }>6 \text { months } \\
\text { and were undergoing general physical } \\
\text { therapy. } \\
\text { Exclusion: } \\
\text { Innate thorax deformity, rib fracture, or had } \\
\text { a disease related to lungs, kidneys, the } \\
\text { endocrine system, orthopedics, or } \\
\text { rheumatology. }\end{array}$ & $\begin{array}{l}\text { Session: IMT with a load of } 30 \% \\
\text { of MIP (adjusted gradually). } 20 \\
\text { min, } 3 \text { times/week, for } 6 \text { weeks. } \\
\text { Device: Respironics }\end{array}$ & Nothing & $\begin{array}{l}-\mathrm{FVC} \\
-\mathrm{FEV1} \\
-\mathrm{PEF}\end{array}$ \\
\hline $\begin{array}{l}\text { Britto et al., } \\
2011 \text { [35] }\end{array}$ & Brazil & $\begin{array}{l}n=21 \\
\text { GI: } 9 \\
\text { GC: } 9 \\
52 \% \text { men } \\
54 \text { years }\end{array}$ & $\begin{array}{l}\text { Inclusion: } \\
\text { Adults }>20 \text { years; hemiparesis due to stroke, } \\
\text { MIP < } 90 \% \text {; no facial palsy, able to use a cycle } \\
\text { ergometer, had no restrictions in lung function } \\
\text { and no neurologic, orthopedic, or unstable } \\
\text { cardiac conditions, nonsmokers, showed no } \\
\text { receptive aphasia, and had not undergone } \\
\text { thoracic or abdominal surgery. } \\
\text { Exclusion: } \\
\text { Patients unable to perform the tests and used } \\
\text { medications that could interfere with } \\
\text { neuromuscular control or cause drowsiness. }\end{array}$ & $\begin{array}{l}\text { Session: IMT } 30 \text { min, } 5 \\
\text { times/week, for } 8 \text { weeks. Each } \\
\text { session was divided into } 6 \\
\text { series of } 5 \text { min each, with a } \\
\text { 1-min rest interval between } \\
\text { series. } \\
\text { IMT with a load of } 30 \% \text { of MIP } \\
\text { (adjusted every } 2 \text { weeks, } \\
\text { according the new MIP value). } \\
\text { Device: Threshold IMT. }\end{array}$ & Sham respiratory training. & -MIP \\
\hline $\begin{array}{l}\text { K Kim et al., } \\
2011 \text { [36] }\end{array}$ & $\begin{array}{l}\text { Korea, } \\
\text { Daegu }\end{array}$ & $\begin{array}{l}n=27 \\
\text { GI: } 13 \\
\text { GC: } 14 \\
\text { 37\% men } \\
57 \text { years }\end{array}$ & $\begin{array}{l}\text { Inclusion: } \\
\text { Stroke occurred greater than } 6 \text { months ago. } \\
\text { Exclusion: } \\
\text { Pulmonary disorders, severe aphasia, and } \\
\text { impairment of cognitive function. }\end{array}$ & $\begin{array}{l}\text { Session: IMT/EMT, } 30 \text { min, } 3 \\
\text { times/week, for } 4 \text { weeks. Load: } \\
50-60 \% \text { of VC and low } \\
\text { frequency (12-13 breaths/ } \\
\text { minute). } \\
\text { Device: SpiroTiger. } \\
\text { RHB program as CG. }\end{array}$ & $\begin{array}{l}\text { Conventional stroke } \\
\text { physical therapy } \\
\text { program ( } 30 \mathrm{~min}, 3 \\
\text { times/week, for } 4 \text { weeks). }\end{array}$ & $\begin{array}{l}-\mathrm{FVC} \\
-\mathrm{FEV1} \\
-\mathrm{PEF}\end{array}$ \\
\hline
\end{tabular}


Table 1. Cont.

\begin{tabular}{|c|c|c|c|c|c|c|}
\hline Study & Country & $\begin{array}{l}\text { Participants/ } \\
\text { Mean Age }\end{array}$ & Inclusion/Exclusion Criteria & Intervention & Control & Study Outcomes \\
\hline $\begin{array}{l}\text { Sutbeyaz et } \\
\text { al., } 2010 \text { [37] }\end{array}$ & Turkey & $\begin{array}{l}n=45 \\
\text { GI1: } 15 \\
\text { GI2: } 15 \\
\text { GC: } 15 \\
53.3 \% \text { men } \\
61.8 \text { years }\end{array}$ & $\begin{array}{l}\text { Inclusion: } \\
\text { Unilateral stroke with hemiparesis in previous } \\
12 \text { months, enough unilateral upper torso and } \\
\text { extremity nerve function and strength to } \\
\text { accomplish arm crank ergometry, ability to } \\
\text { understand and follow simple verbal } \\
\text { instructions, no previous history of } \\
\text { cardiovascular or respiratory problems, no } \\
\text { medication that would influence metabolic or } \\
\text { cardiorespiratory responses to exercise. } \\
\text { Exclusion: } \\
\text { Chronic pulmonary and/or cardiac disease, } \\
\text { clinical signs of cardiac and/or respiratory } \\
\text { disease, impaired level of consciousness and } \\
\text { evidence of gross cognitive impairment. }\end{array}$ & $\begin{array}{l}\text { Session: } 2 \text { sets/day of } 15 \mathrm{~min} \\
\text { each, six times a week, for } 6 \\
\text { weeks. } \\
\text { Load of IMT starting at a load } \\
\text { of } 40 \% \text { MIP. It was gradually } \\
\text { increased, } 5-10 \% \text { each session, } \\
\text { to } 60 \% \text { of MIP as tolerated. } \\
\text { Device: Threshold IMT. } \\
\text { RHB program as CG. }\end{array}$ & $\begin{array}{l}\text { Conventional stroke } \\
\text { rehabilitation program, } 5 \\
\text { days a week for } 6 \text { weeks. }\end{array}$ & $\begin{array}{l}\text {-FVC } \\
\text {-FEV1 } \\
\text {-PEF } \\
\text {-MIP } \\
\text {-MEP } \\
\text {-Dyspnea }\end{array}$ \\
\hline
\end{tabular}

IME, inspiratory muscle endurance; MIP, maximal inspiratory pressure; MEP, maximal expiratory pressure; CG, control group; IG, intervention group; NIHSS, National Institutes of Health Stroke Scale; RHB, rehabilitation; MBI, Barthel Index; BBS, Berg Balance Scale; K-MMSE, Korean Mini-Mental State Examination, IMT, inspiratory muscle training; EMT, expiratory muscle training; 6-MWT, 6 min walking test; NA, not available; RMT; respiratory muscle training; FVC, forced vital capacity; PEF: peak expiratory flow; TIS, trunk impaired scale; VC, vital capacity; MRS, Modified Rankin Scale; FEV1, first second forced expiratory volume. 


\subsection{Risk of Bias Assessment}

The risk of bias of included studies was assessed using the PEDro Scale. All RCTs included complied with the following items: random allocation, to report between-group differences and in to report point estimate and variability (Table 2). The highest score was eight points for two studies [21,30]. Four studies scored seven $[19,31,35,37]$, mainly due to the non-blinding of therapist and assessors and the non-presentation of an intention-to-treat analysis. Five studies scored six and four studies obtained five points. The lowest score was three points for the study of the NJ Jung et al. [25]. The three resting studies obtained four points $[24,33,34]$. Thus, fifteen studies obtained higher or equal to five points, a moderate quality.

\subsection{Effect of Intervention: Pooled Estimates}

\subsubsection{Pulmonary Function}

Among nineteen studies included, eleven displayed results about FEV1 [19,20,23,25,27,29,32-34, 36,37]. When all RMT interventions (IMT/EMT and IMT) were jointly analysed, the summary ES was 0.57 (95\% CI: 0.24 to $0.90 ; \mathrm{I}^{2}=42.9 \%, p=0.064$ ) (Figure 2 ). The subgroup analysis by type of intervention showed a slightly greater effect in the pooled of the studies that performed only IMT [ES= 0.89 (95\% CI: 0.19 to $\left.1.58 ; \mathrm{I}^{2}=66.3 \%, p=0.018\right)$ ] compared with the IMT/EMT subgroup [ES=0.38 $(95 \%$ CI: 0.06 to $\left.\left.0.69 ; \mathrm{I}^{2}=0.0 \%, p=0.650\right)\right]$.

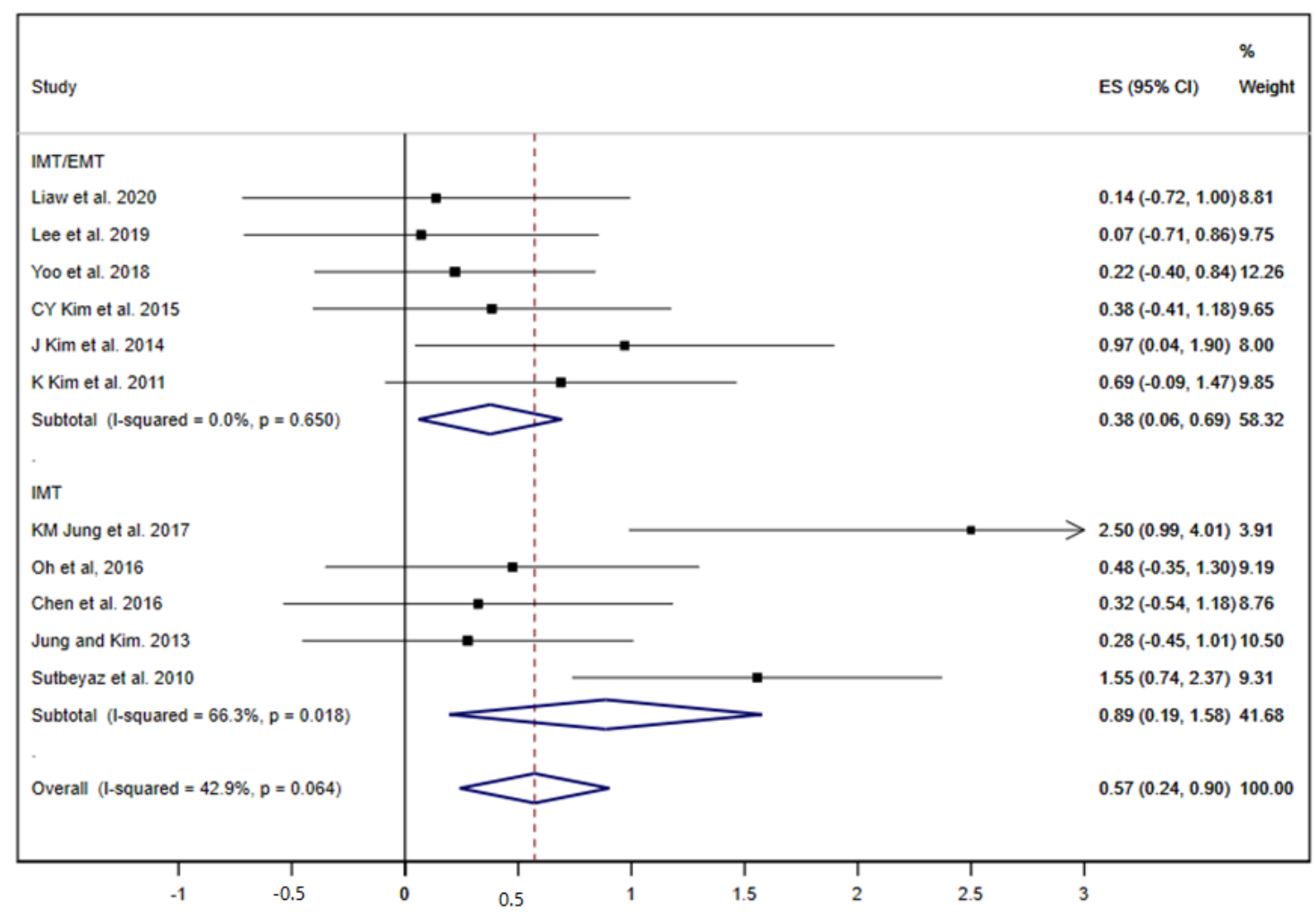

Figure 2. Forest plot showing the effect size (ES) of respiratory muscle training (RMT) on first second forced expiratory volume (FEV1) between intervention and control groups for each study. IMT, inspiratory muscle training; EMT, expiratory muscle training. 
Table 2. Risk of bias and study quality on the PEDro Scale.

\begin{tabular}{|c|c|c|c|c|c|c|c|c|c|c|c|}
\hline Study & $\begin{array}{c}\text { Random } \\
\text { Allocation }\end{array}$ & $\begin{array}{l}\text { Concealed } \\
\text { Allocation }\end{array}$ & $\begin{array}{l}\text { Baseline } \\
\text { Similarity }\end{array}$ & $\begin{array}{c}\text { Subject } \\
\text { Blinding }\end{array}$ & $\begin{array}{l}\text { Therapist } \\
\text { Blinding }\end{array}$ & $\begin{array}{l}\text { Assessor } \\
\text { Blinding }\end{array}$ & $\begin{array}{c}<15 \% \\
\text { Dropouts }\end{array}$ & $\begin{array}{c}\text { Intention to-Treat } \\
\text { Analysis }\end{array}$ & $\begin{array}{c}\text { Between-Group } \\
\text { Difference Reported }\end{array}$ & $\begin{array}{c}\text { Point Estimate, } \\
\text { Variability Reported }\end{array}$ & Total \\
\hline Liaw et al., 2020 [19] & $\mathrm{Y}$ & $\mathrm{Y}$ & $\mathrm{Y}$ & $\mathrm{N}$ & $\mathrm{Y}$ & $\mathrm{N}$ & $\mathrm{N}$ & $\mathrm{Y}$ & $\mathrm{Y}$ & $\mathrm{Y}$ & 7 \\
\hline Lee et al., 2019 [20] & $\mathrm{Y}$ & $\mathrm{Y}$ & $\mathrm{Y}$ & $\mathrm{N}$ & $\mathrm{N}$ & $\mathrm{Y}$ & $\mathrm{N}$ & $\mathrm{N}$ & $Y$ & $Y$ & 6 \\
\hline Menezes et al., 2019 [21] & $\mathrm{Y}$ & $\mathrm{Y}$ & $\mathrm{Y}$ & $\mathrm{Y}$ & $\mathrm{N}$ & $\mathrm{N}$ & $\mathrm{Y}$ & $\mathrm{Y}$ & $Y$ & $\mathrm{Y}$ & 8 \\
\hline Cho et al., 2018 [22] & Y & Y & Y & $\mathrm{N}$ & $\mathrm{N}$ & $\mathrm{Y}$ & $\mathrm{N}$ & $\mathrm{N}$ & $\mathrm{Y}$ & Y & 6 \\
\hline Yoo et al., 2018 [23] & $\mathrm{Y}$ & $\mathrm{N}$ & $\mathrm{Y}$ & $\mathrm{N}$ & $\mathrm{N}$ & $\mathrm{N}$ & $\mathrm{Y}$ & $\mathrm{N}$ & $\mathrm{Y}$ & $Y$ & 5 \\
\hline Lee et al., 2018 [24] & $\mathrm{Y}$ & $\mathrm{N}$ & $\mathrm{Y}$ & $\mathrm{N}$ & $\mathrm{N}$ & $\mathrm{N}$ & $\mathrm{N}$ & $\mathrm{N}$ & $\mathrm{Y}$ & $\mathrm{Y}$ & 4 \\
\hline KM Jung et al., 2017 [25] & $\mathrm{Y}$ & $\mathrm{Y}$ & $\mathrm{Y}$ & $\mathrm{N}$ & $\mathrm{N}$ & $\mathrm{N}$ & $\mathrm{Y}$ & $\mathrm{N}$ & $\mathrm{Y}$ & $\mathrm{Y}$ & 6 \\
\hline NJ Jung et al., 2017 [26] & $\mathrm{Y}$ & $\mathrm{N}$ & $\mathrm{N}$ & $\mathrm{N}$ & $\mathrm{N}$ & $\mathrm{N}$ & $\mathrm{N}$ & $\mathrm{N}$ & $Y$ & $Y$ & 3 \\
\hline Guillen-Sola et al., 2017 [28] & $\mathrm{Y}$ & $\mathrm{N}$ & $\mathrm{Y}$ & $\mathrm{N}$ & $\mathrm{N}$ & $\mathrm{Y}$ & $\mathrm{N}$ & $\mathrm{Y}$ & $\mathrm{Y}$ & $\mathrm{Y}$ & 6 \\
\hline Oh et al., 2016 [27] & $\mathrm{Y}$ & $\mathrm{N}$ & $\mathrm{Y}$ & $\mathrm{N}$ & $\mathrm{N}$ & $\mathrm{N}$ & $\mathrm{Y}$ & $\mathrm{N}$ & $\mathrm{Y}$ & $\mathrm{Y}$ & 5 \\
\hline Chen et al., 2016 [29] & $\mathrm{Y}$ & $\mathrm{N}$ & Y & $\mathrm{N}$ & $\mathrm{N}$ & Y & $\mathrm{N}$ & $\mathrm{N}$ & Y & Y & 5 \\
\hline Messagi-Sartor et al., 2015 [30] & $\mathrm{Y}$ & $\mathrm{Y}$ & $\mathrm{Y}$ & $\mathrm{Y}$ & $\mathrm{N}$ & $\mathrm{Y}$ & $\mathrm{N}$ & $\mathrm{Y}$ & $\mathrm{Y}$ & $\mathrm{Y}$ & 8 \\
\hline Kulnik et al., 2015 [31] & $\mathrm{Y}$ & $\mathrm{Y}$ & $\mathrm{Y}$ & $\mathrm{N}$ & $\mathrm{N}$ & $\mathrm{Y}$ & $\mathrm{N}$ & $\mathrm{Y}$ & $\mathrm{Y}$ & $\mathrm{Y}$ & 7 \\
\hline CY Kim et al., 2015 [32] & $\mathrm{Y}$ & $\mathrm{N}$ & $\mathrm{Y}$ & $\mathrm{N}$ & $\mathrm{N}$ & $\mathrm{Y}$ & $\mathrm{N}$ & $\mathrm{N}$ & $\mathrm{Y}$ & Y & 5 \\
\hline J Kim et al., 2014 [33] & $\mathrm{Y}$ & $\mathrm{N}$ & $\mathrm{Y}$ & $\mathrm{N}$ & $\mathrm{N}$ & $\mathrm{N}$ & $\mathrm{N}$ & $\mathrm{N}$ & $Y$ & $Y$ & 4 \\
\hline Jung and Kim 2013 [34] & $\mathrm{Y}$ & $\mathrm{N}$ & $\mathrm{Y}$ & $\mathrm{N}$ & $\mathrm{N}$ & $\mathrm{N}$ & $\mathrm{N}$ & $\mathrm{N}$ & $Y$ & $\mathrm{Y}$ & 4 \\
\hline Britto et al., 2011 [35] & $\mathrm{Y}$ & $\mathrm{Y}$ & $\mathrm{Y}$ & $\mathrm{N}$ & $\mathrm{N}$ & $\mathrm{Y}$ & $\mathrm{Y}$ & $\mathrm{N}$ & $\mathrm{Y}$ & $\mathrm{Y}$ & 7 \\
\hline K Kim et al., 2011 [36] & $\mathrm{Y}$ & $\mathrm{N}$ & $\mathrm{Y}$ & $\mathrm{Y}$ & $\mathrm{N}$ & $\mathrm{Y}$ & $\mathrm{N}$ & $\mathrm{N}$ & $\mathrm{Y}$ & $\mathrm{Y}$ & 6 \\
\hline Sutbeyaz et al., 2010 [37] & $\mathrm{Y}$ & $\mathrm{Y}$ & $\mathrm{Y}$ & $\mathrm{N}$ & $\mathrm{N}$ & $\mathrm{Y}$ & $\mathrm{Y}$ & $\mathrm{N}$ & $\mathrm{Y}$ & $\mathrm{Y}$ & 7 \\
\hline
\end{tabular}


The FVC was analysed in nine studies $[19,23,25,27,29,32,34,36,37]$. The pooled ES was $0.32(95 \%$ CI: 0.06 to $0.58 ; \mathrm{I}^{2}=0.0 \%, p=0.507$ ) (Figure 3 ). The subgroup analysis by type of RMT exercises did not show great differences between the ES of both groups, although without statistical significance in any group (IMT and IMT/EMT).

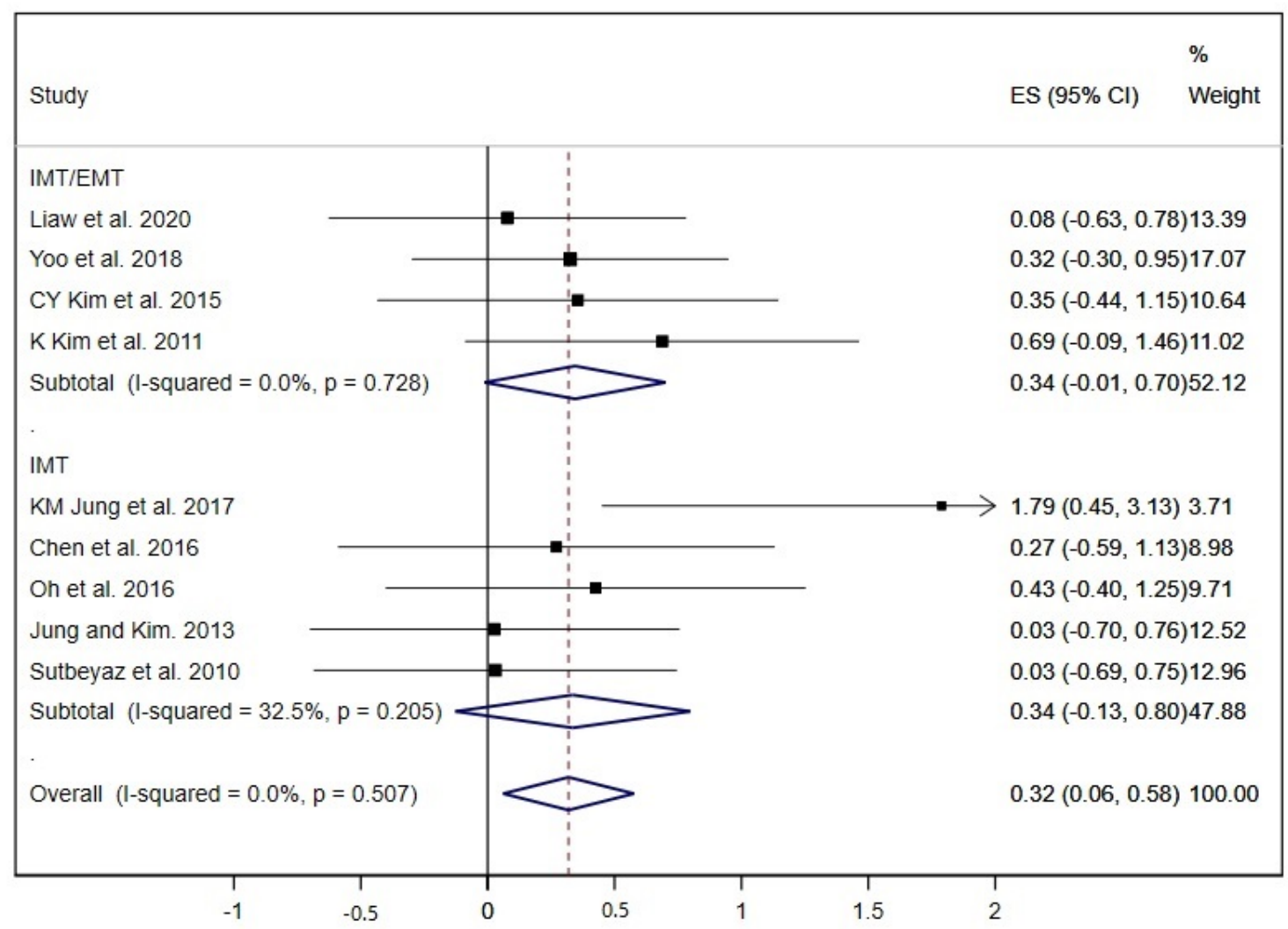

Figure 3. Forest plot showing the effect size (ES) of respiratory muscle training (RMT) on forced vital capacity (FVC) between intervention and control groups for each study. IMT, inspiratory muscle training; EMT, expiratory muscle training.

Other pulmonary function parameters such as PEF and the strength of inspiratory and expiratory muscles (MIP and MEP) were analysed in the studies included. Seven studies showed results about PEF and the pooled estimated ES was 0.48 (95\% CI: 0.20 to $0.77 ; \mathrm{I}^{2}=0.0 \%, p=0.823$ ) (Figure 4) [20, $23,27,33,34,36,37]$, nevertheless, the subgroup analysis displayed a statistically significant ES in the IMT/EMT group (ES $=0.55 ; 95 \%$ CI: 0.17 to 0.92 ) but not in the IMT group (ES $=0.40 ; 95 \%$ CI: -0.04 to 0.84 ).

Equally, MEP was analysed in seven studies [19-21,28,30,31,37], and only one of these performed exclusively IMT exercises [37]; thus, the overall ES was 0.55 ( $95 \%$ CI: 0.12 to $0.98 ; \mathrm{I}^{2}=71.9 \%, p=0.002$ ) (Figure 5). 


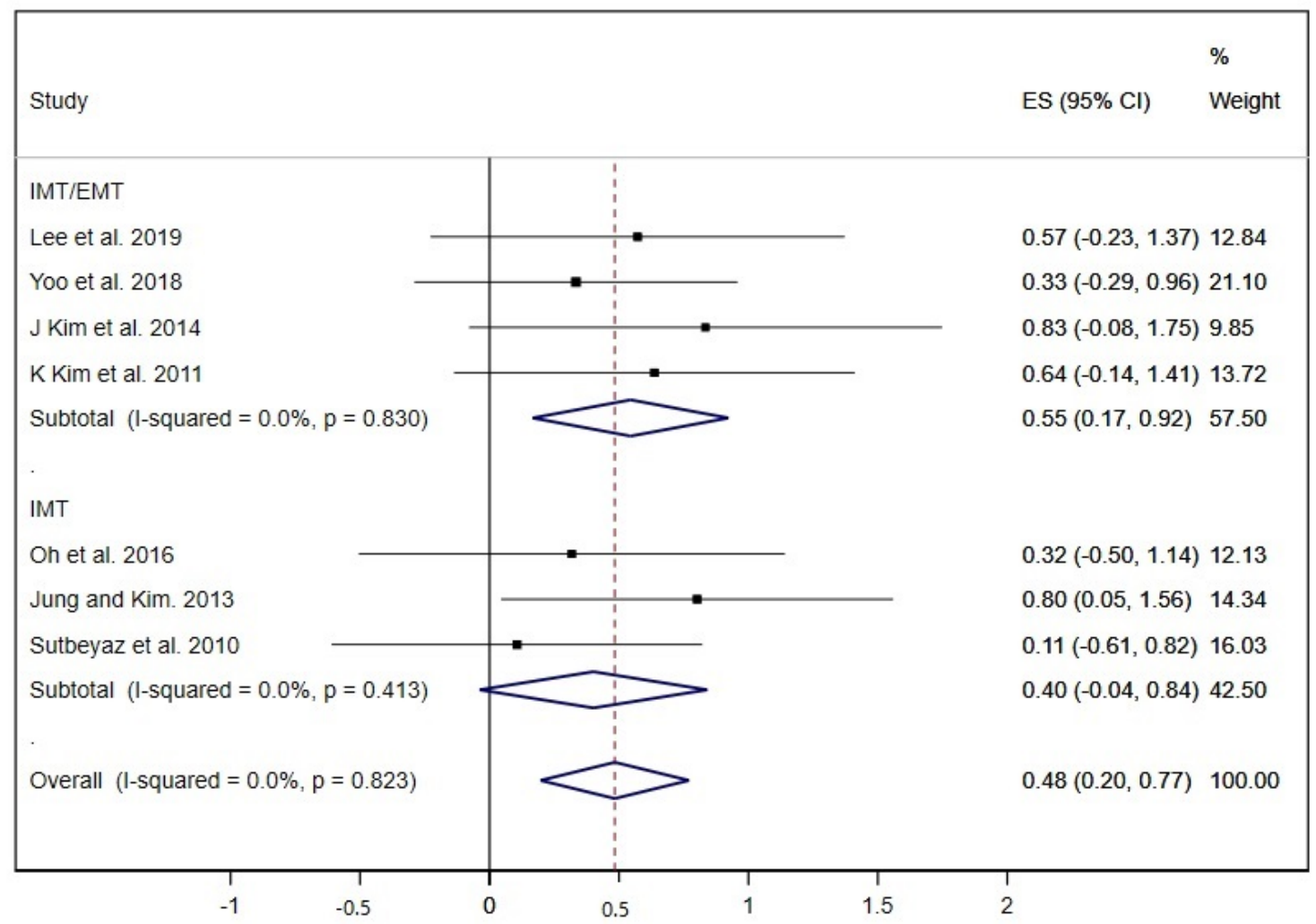

Figure 4. Forest plot showing the effect size (ES) of respiratory muscle training (RMT) on peak expiratory flow (PEF) between intervention and control groups for each study. IMT, inspiratory muscle training; EMT, expiratory muscle training.

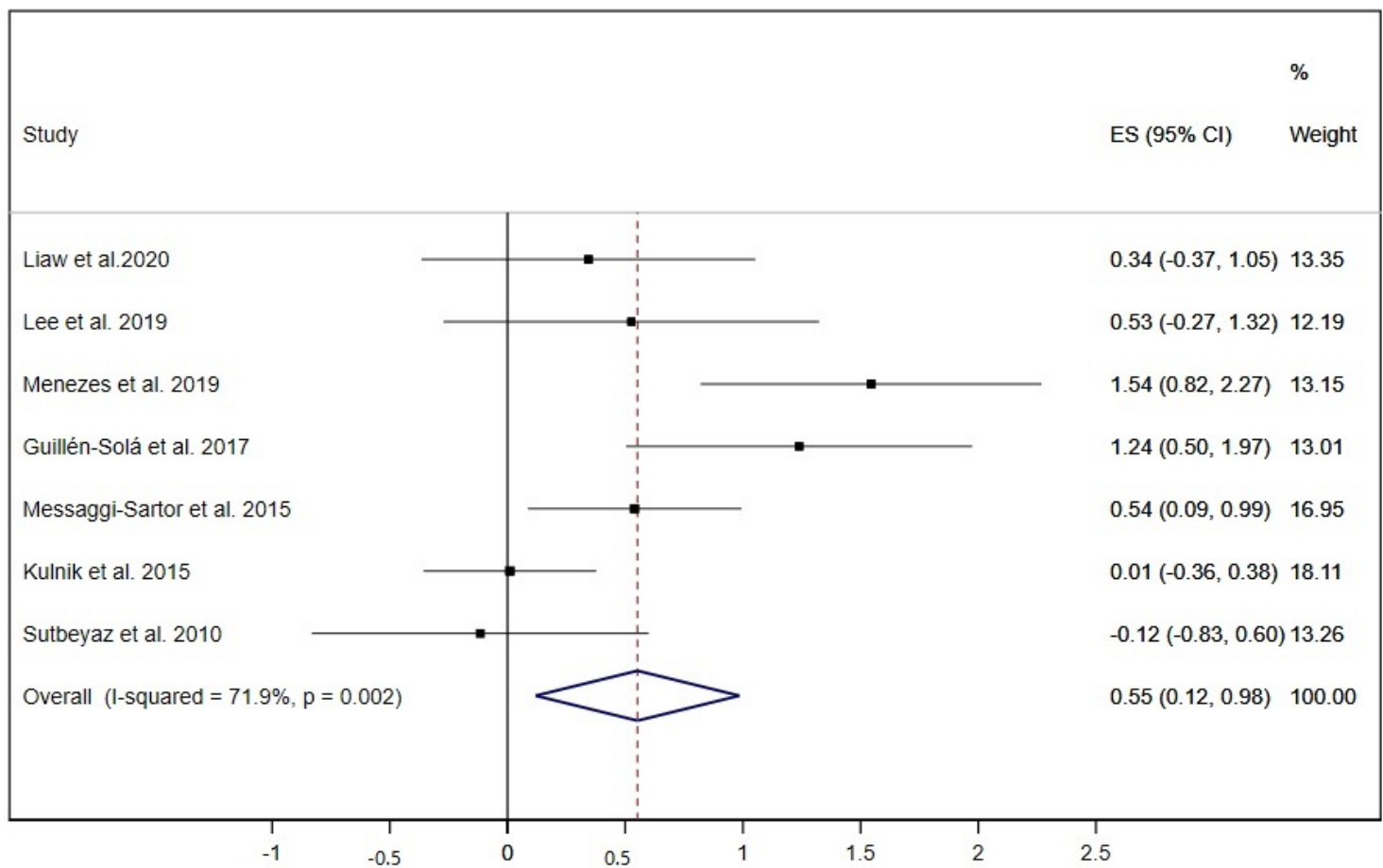

Figure 5. Forest plot showing the effect size (ES) of respiratory muscle training (RMT) on maximal expiratory pressure (MEP) between intervention and control groups for each study. IMT, inspiratory muscle training; EMT, expiratory muscle training. 
The MIP was examined in nine studies [19-22,28,30,31,35,37], and obtained a pooled ES $=0.84$ (95\% CI: 0.44 to $1.24 ; \mathrm{I}^{2}=69.2 \%, p=0.001$ ) (Figure 6); three of these studies performed only IMT exercises [22,35,37], and six studies carried out both IMT and EMT exercises, showing statistically significant improvements for both groups of interventions.

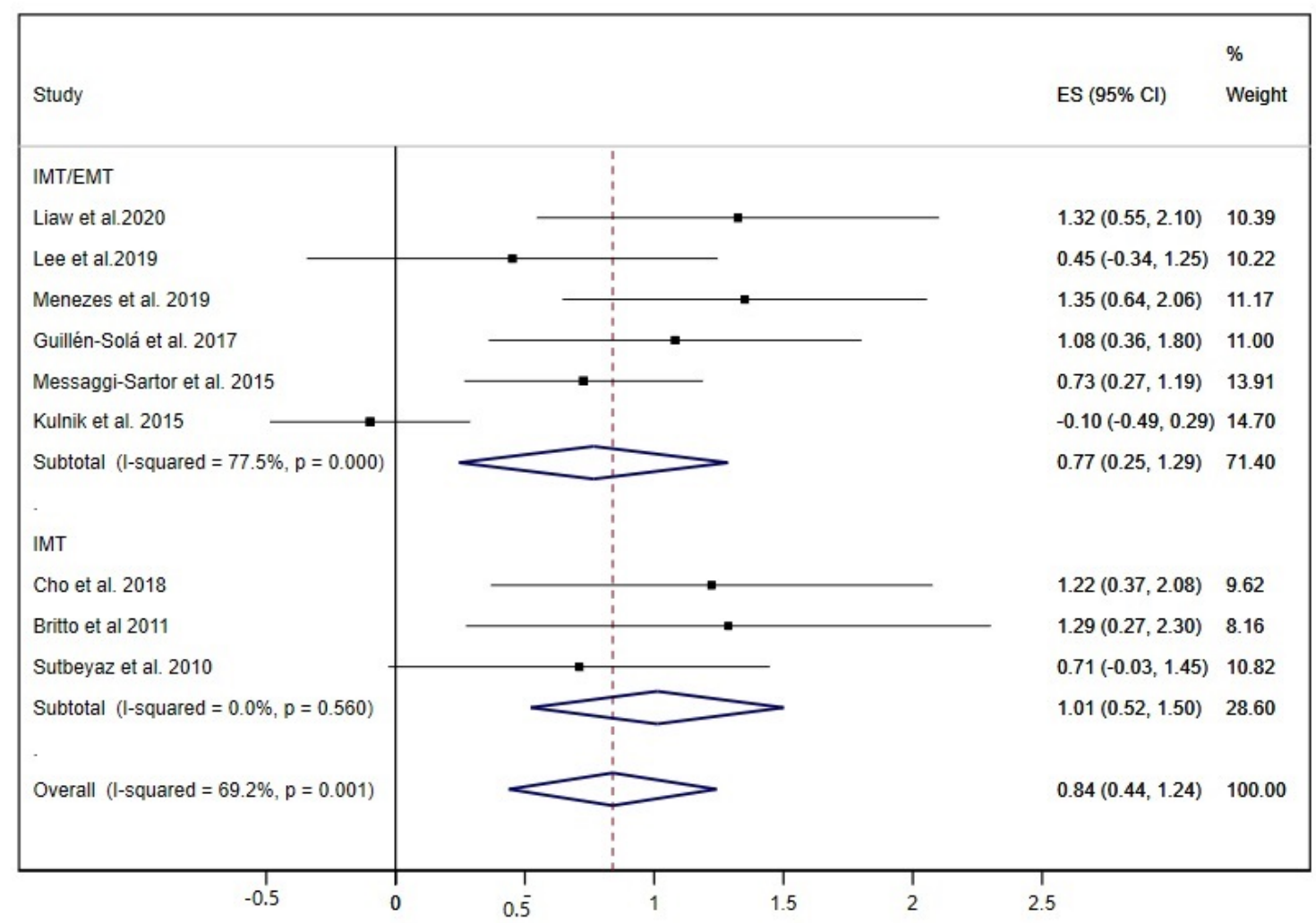

Figure 6. Forest plot showing the effect size (ES) of respiratory muscle training (RMT) on maximal inspiratory pressure (MIP) between intervention and control groups for each study. IMT, inspiratory muscle training; EMT, expiratory muscle training.

Sensitivity analyses for all pulmonary function variables showed that the pooled ES estimates were not significantly modified in magnitude or direction when individual study data were removed from the analysis one at a time. The exception was the FVC analysis, which lost the statistical significance when the studies of KM Jung et al. [25] and K Kim et al. [36] were removed from the analysis.

There was no evidence of publication bias by funnel plot asymmetry and Egger's test in all pulmonary function variables studied.

\subsubsection{Functional Capacity}

Six out of nineteen studies evaluated the 6-MWT [21,22,24-26,33]. Of these, two studies only performed IMT exercises [25,26]; for this, a subgroup analysis by type of RMT exercises was not performed. The overall ES for the 6-MWT was 0.39 (95\% CI: 0.05 to $0.74 ; \mathrm{I}^{2}=0.0 \%, p=0.919$ ) (Figure 7). When the studies were removed one by one, the pooled ES analysis showed a loss of statistical significance when the study of NJ Jung et al. [26] was removed. 


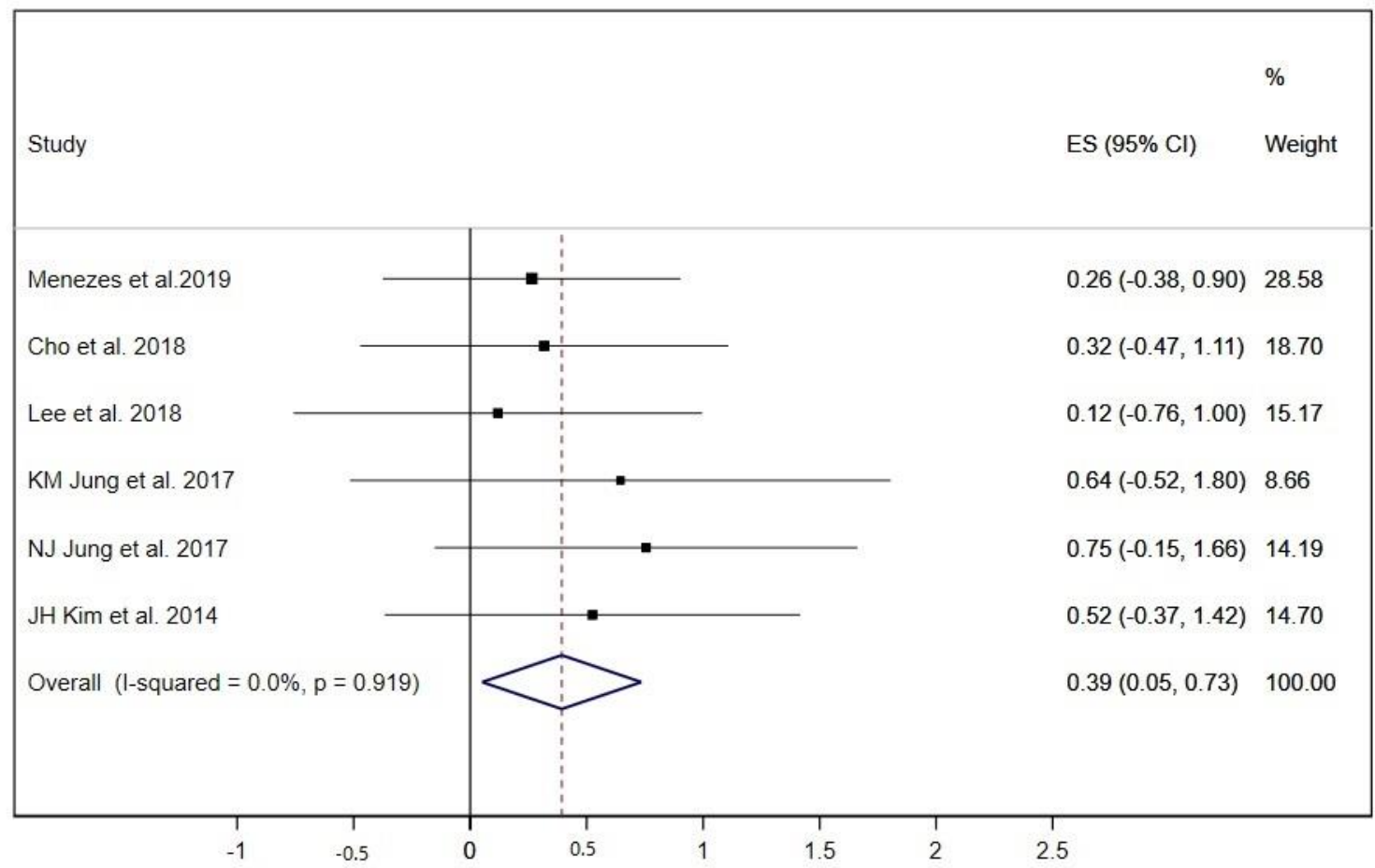

Figure 7. Forest plot showing the effect size (ES) of respiratory muscle training (RMT) on 6 min walking test (6-MWT) between intervention and control groups for each study. IMT, inspiratory muscle training; EMT, expiratory muscle training.

Dyspnea was evaluated in five studies $[19,21,29,33,37]$; due to the limited number of studies, the subgroup analysis by type of RMT exercise was not performed. The pooled ES of RMT interventions on dyspnea was 0.47 (95\% CI: -0.30 to $\left.1.23 ; \mathrm{I}^{2}=77 \%, p=0.002\right)$ (Figure 8 ), showing a positive trend of improvement which was not statistically significant.

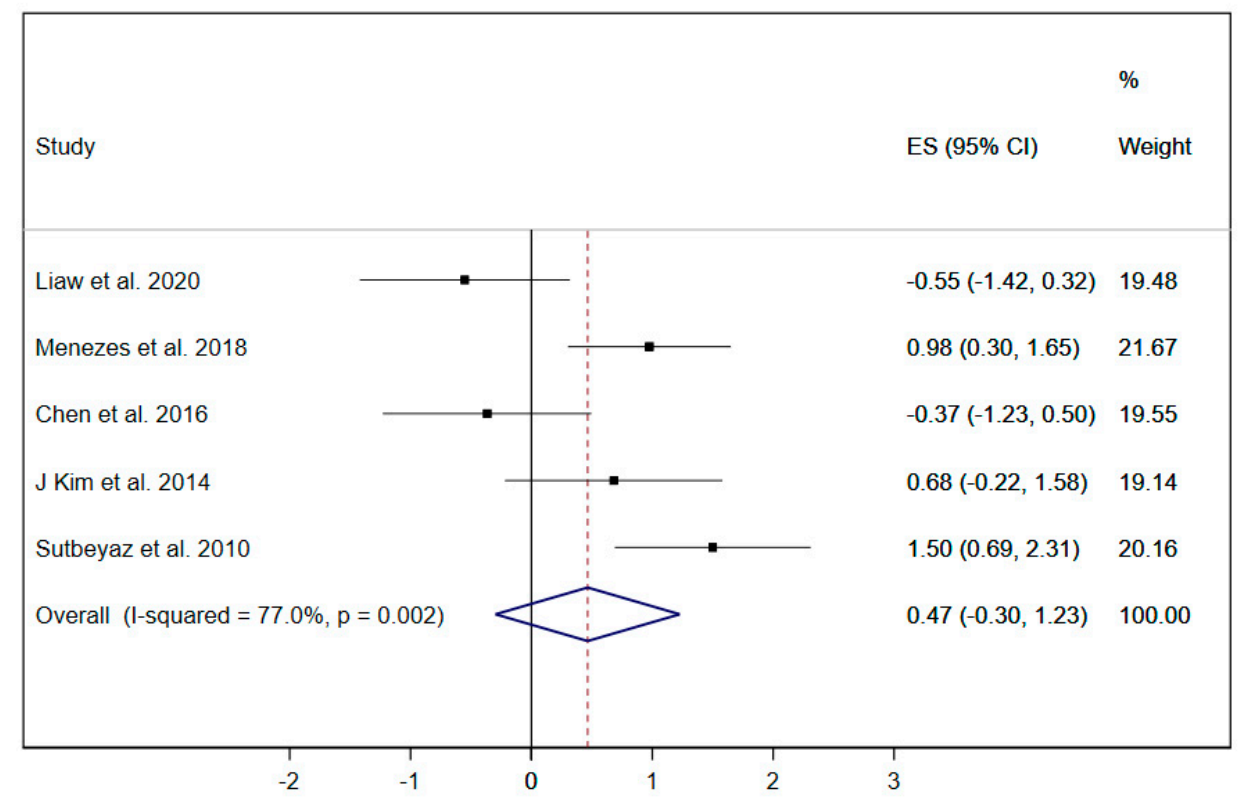

Figure 8. Forest plot showing the effect size (ES) of respiratory muscle training (RMT) on dyspnea between intervention and control groups for each study. IMT, inspiratory muscle training; EMT, expiratory muscle training. 
The MBI $[19,23,29]$ and BBS $[23,24,27]$ were only evaluated in three studies. In both, the RMT interventions did not show a statistically significant improvement (MBI: 0.22 (95\% CI: -0.31 to 0.75 ; $\mathrm{I}^{2}=37.6 \%, p=0.201$; and BBS: 0.06 (95\% CI: -0.37 to $0.49 ; \mathrm{I}^{2}=0.0 \%, p=0.953$ ).

Publication bias was not assessed by funnel plot and Egger test, due to the reduced number of studies that evaluated these variables of functional capacity [38].

\section{Discussion}

In the present study, the results show a significant positive relationship between the RMT interventions on the improvement of pulmonary function parameters (FEV1, FVC, PEF, MEP, MIP) and the functional capacity such as walking ability (6-MWT) but not in balance (BBS), MBI and dyspnea, in patients with stroke.

The subgroup analyses by type of RMT exercises did not show differences between the exclusive IMT interventions and the IMT/EMT interventions, except in the PEF, in which the combined IMT and EMT exercises showed a greater effect than only IMT exercises on the improvements of this pulmonary parameter.

A previously published meta-analysis that included eleven trials also obtained similar results [7]. Nevertheless, the main difference was found in dyspnea. Contrary to this study, our meta-analysis did not find a positive effect of RMT on dyspnea in patients with stroke. These differences could be due to several reasons: first, the previous study only included two moderate methodological quality RCTs, a reduced number of studies to provide consistent conclusions about the effectiveness of RMT on dyspnea; second, patients with stroke usually have a low perception of dyspnea, due to their dissociation between respiratory effort and dyspnea [39].

Regarding the pulmonary function parameters, our results showed positive effectiveness of RMT interventions to improve FEV1, FVC, PEF, MEP, and MIP in patients with stroke. These results are in line with previous systematic reviews and meta-analyses results $[7,8]$. Patients who have suffered a stroke present abdominal and diaphragm dysfunction, causing a decrease of respiratory muscle strength $[2,40,41]$. This weakness of respiratory muscles is usually associated with reduced lung volumes, flows and restrictive ventilatory patterns [42]. Thus, it seems obvious, and the results of this meta-analysis corroborate this, that the training of the respiratory muscle will improve the lung volumes and flows, noting an increase of FEV1, FVC, PEF, MEP and MIP.

Aiming to facilitate the clinical interpretation of these results, we expressed the pooled effect estimate of each pulmonary function parameter on clinical measurement improvements, using methods recommended by the Cochrane Collaboration [43]. Thus, our data show that RMT interventions increase baseline FEV1 values in $12.2 \%$ of predicted-FEV1 by age and sex, FVC baseline values improve in $6.75 \%$ of predicted FVC, PEF baseline measure increases in 46.97 litres per second. Finally, MEP and MIP improved their baseline values in 10.05 and $22.40 \mathrm{~cm} \mathrm{H}_{2} 0$, respectively. These results align with previously published meta-analysis [7-10].

The improvement of inspiratory and expiratory muscle strength had significant implications for the functional capacity of poststroke patients. The weakness of respiratory muscles, secondary to stroke, can lead to a reduced tolerance to exercise $[8,44]$. Thus, the benefits gained by the increase of respiratory muscle strength and lung function could improve the tolerance to exercise [8].

Our results showed that RMT achieved an improvement of walking ability measured by 6-MWT, with an ES=0.39, which when translated in terms of clinical measure improvement, using methods endorsed by the Cochrane Collaboration [43], is equal to an increase of $25 \mathrm{~m}$ travelled in $6 \mathrm{~min}$. Although it could be seen as little progress, walking ability is a significant predictor of physical activity and social and community participation after stroke $[45,46]$, so even small improvements could imply immense benefits for the physical and social health of stroke patients. They can help these patients to carry out daily activities more efficiently.

In line with this, three studies reported results about the Barthel index, a scale used to measure disability or dependence in activities of daily living [47]. The pooled estimate showed a positive but 
not statistically significant trend. The same occurred with the BBS [48]. Nevertheless, the limited number of studies that reported these variables do not allow for robust conclusions about it.

Despite the wide variety of characteristics of RMT interventions, the previous meta-analysis concluded that $30 \mathrm{~min}$ of RMT, five times/week, for five weeks could be sufficient to increase respiratory muscle strength in very weak poststroke patients [3].

This study has several limitations that must be considered, some of them, such as the heterogeneity, are inherent to meta-analyses. First, although a higher number of studies than the previous meta-analysis was included, not all of them report the same variables, so some variables such as MBI or BBS only were informed in three studies. Second, it is known that patients with more significant respiratory muscle weakness usually respond better to RMT [8], and in the present work around the half studies [19-22,28-31,37] included poststroke patients with respiratory muscle weakness (MIP $<50 \mathrm{~cm}$ $\mathrm{H}_{2} \mathrm{O}$ ) which could have influenced the results. Third, the inclusion/exclusion criteria were different in the studies included, equally to the RMT characteristics of interventions. Nevertheless, the subgroup analysis by type of RMT exercises allowed for the comparison of the effect of IMT alone with the IMT and EMT exercises. Fourth, despite the number of studies included, the total sample of participants included was scarce.

\section{Conclusions}

The RMT interventions are effective in improving pulmonary function parameters (FEV1, FVC, PEF), strength of expiratory and inspiratory muscles (MEP and MIP), and walking ability in poststroke patients. More well-designed RCTs with larger sample sizes are needed to examine the most appropriate features of interventions: IMT, EMT or both, duration, frequency, and intensity to establish the highest clinical efficacy.

Supplementary Materials: The following are available online at http://www.mdpi.com/1660-4601/17/15/5356/s1, File S1: Articles excluded and reasons.

Author Contributions: Conceptualization, D.P.P.-C. and A.I.C.-C.; methodology, D.P.P.-C. and A.I.C.-C.; software, D.P.P.-C.; validation, D.P.P.-C., J.A.L.-A., J.M.C.-T. and A.I.C.-C.; formal analysis, D.P.P.-C. and A.I.C.-C.; data curation, D.P.P.-C. and A.I.C.-C.; Writing-Original Draft preparation, D.P.P.-C., and A.I.C.-C.; Writing-Review and Editing, D.P.P.-C., J.M.C.-T., J.A.L.-A., P.Á.L.-R., J.A.P.-M. and A.I.C.-C.; visualization, D.P.P.-C., J.M.C.-T., J.A.L.-A., P.Á.L.-R., J.A.P.-M. and A.I.C.-C.; supervision, D.P.P.-C.; project administration, D.P.P.-C. All authors have read and agreed to the published version of the manuscript.

Funding: Diana P. Pozuelo Carrascosa is contracted by FEDER funds (2019/7375). This research was funded by a grant from the European Regional Development Fund (ERDF) [Fondo Europeo de Desarrollo Regional (FEDER) (DOCM 19/02/20)].

Conflicts of Interest: The authors have declared no conflict of interest. The funders had no role in the design of the study, in the collection, analyses, or interpretation of data; in the writing of the manuscript, or in the decision to publish the results.

\section{References}

1. Hankey, G.J. Stroke. Lancet 2017, 389, 641-654. [CrossRef]

2. Jandt, S.R.; Caballero, R.M.D.S.; Junior, L.A.F.; Dias, A.S. Correlation between trunk control, respiratory muscle strength and spirometry in patients with stroke: An observational study. Physiother. Res. Int. 2010, 16, 218-224. [CrossRef] [PubMed]

3. Menezes, K.K.P.; Nascimento, L.R.; Ada, L.; Polese, J.C.; Avelino, P.R.; Teixeira-Salmela, L.F. Respiratory muscle training increases respiratory muscle strength and reduces respiratory complications after stroke: A systematic review. J. Physiother. 2016, 62, 138-144. [CrossRef]

4. Polese, J.C.; Pinheiro, M.B.; Faria, C.D.C.M.; Britto, R.R.; Parreira, V.F.; Teixeira-Salmela, L.F. Strength of the respiratory and lower limb muscles and functional capacity in chronic stroke survivors with different physical activity levels. Braz. J. Phys. Ther. 2013, 17, 487-493. [CrossRef] [PubMed] 
5. Battaglia, E.; Fulgenzi, A.; Ferrero, M.E. Rationale of the combined use of inspiratory and expiratory devices in improving maximal inspiratory pressure and maximal expiratory pressure of patients with chronic obstructive pulmonary disease. Arch. Phys. Med. Rehabil. 2009, 90, 913-918. [CrossRef] [PubMed]

6. Forbes, S.; Game, A.; Syrotuik, D.; Jones, R.; Bell, G.J. The effect of inspiratory and expiratory respiratory muscle training in rowers. Res. Sports Med. 2011, 19, 217-230. [CrossRef] [PubMed]

7. De Menezes, K.K.P.; Nascimento, L.R.; Avelino, P.R.; Alvarenga, M.T.M.; Teixeira-Salmela, L.F. Efficacy of interventions to improve respiratory function after stroke. Respir. Care 2018, 63, 920-933. [CrossRef]

8. Gomes-Neto, M.; Saquetto, M.B.; Silva, C.M.; Carvalho, V.O.; Ribeiro, N.; Conceição, C.S. Effects of respiratory muscle training on respiratory function, respiratory muscle strength and exercise tolerance in post-stroke patients: A systematic review with meta-analysis. Arch. Phys. Med. Rehabil. 2016, 97, 1994-2001. [CrossRef]

9. Xiao, Y.; Luo, M.; Wang, J.; Luo, H. Inspiratory muscle training for the recovery of function after stroke (Cochrane review). Cochrane Database Syst. Rev. 2012, 5. [CrossRef]

10. Martín-Valero, R.; Almeida, M.D.L.C.; Casuso-Holgado, M.J.; Heredia-Madrazo, A. Systematic Review of Inspiratory Muscle Training after Cerebrovascular Accident. Respir. Care 2015, 60, 1652-1659. [CrossRef]

11. Moher, D.; Liberati, A.; Tetzlaff, J.; Altman, D.G. Preferred reporting items for systematic reviews and meta-analyses: The PRISMA statement. PLoS Med. 2009, 6, e1000097. [CrossRef] [PubMed]

12. Higgins, J.P.T.; Thomas, J.; Chandler, J.; Cumpston, M.; Li, T.; Page, M.J.; Welch, V.A. (Eds.) Cochrane Handbook for Systematic Reviews of Interventions, 2nd ed.; John Wiley \& Sons: Chichester, UK, 2019.

13. Higgins, J.P.; Thomas, S.J.; Page, M.J.; Elbers, R.G.; Sterne, J.A.C. Chapter 8: Assessing risk of bias in a randomized trial. In Cochrane Handbook for Systematic Reviews of Interventions Version 60 (Updated July 2019): Cochrane; Higgins, J.P., Thomas, J., Chandler, J., Cumpston, M., Li, T., Page, M.J., Welch, V.A., Eds.; John Wiley \& Sons: Chichester, UK, 2019.

14. Olivo, S.A.; Macedo, L.; Gadotti, I.C.; Fuentes, J.; Stanton, T.R.; Magee, D.J. Scales to Assess the Quality of Randomized Controlled Trials: A Systematic Review. Phys. Ther. 2008, 88, 156-175. [CrossRef] [PubMed]

15. Maher, C.G.; Sherrington, C.; Herbert, R.D.; Moseley, A.M.; Elkins, M.R. Reliability of the PEDro Scale for Rating Quality of Randomized Controlled Trials. Phys. Ther. 2003, 83, 713-721. [CrossRef] [PubMed]

16. Morris, S.B. Estimating Effect Sizes From Pretest-Posttest-Control Group Designs. Organ. Res. Methods 2007, 11, 364-386. [CrossRef]

17. DerSimonian, R.; Laird, N. Meta-analysis in clinical trials. Control. Clin. Trials 1986, 7, 177-188. [CrossRef]

18. Higgins, J.P.T.; Li, T.; Deeks, J.J. Chapter 6: Choosing effect measures and computing estimates of effect. In Cochrane Handbook for Systematic Reviews of Interventions Version 60 (Updated July 2019): Cochrane; Higgins, J.P., Thomas, J., Chandler, J., Cumpston, M., Li, T., Page, M.J., Welch, V.A., Eds.; John Wiley \& Sons: Chichester, UK, 2019.

19. Liaw, M.Y.; Hsu, C.H.; Leong, C.P.; Liao, C.Y.; Wang, L.Y.; Lu, C.H.; Lin, M.C. Respiratory muscle training in stroke patients with respiratory muscle weakness, dysphagia, and dysarthria-A prospective randomized trial. Medicine (Baltimore) 2020, 99, e19337. [CrossRef]

20. Lee, K.; Park, D.; Lee, G. Progressive Respiratory Muscle Training for Improving Trunk Stability in Chronic Stroke Survivors: A Pilot Randomized Controlled Trial. J. Stroke Cerebrovasc. Dis. 2019, 28, 1200-1211. [CrossRef]

21. De Menezes, K.K.P.; Nascimento, L.R.; Ada, L.; Avelino, P.R.; Polese, J.C.; Alvarenga, M.T.M.; Barbosa, M.H.; Teixeira-Salmela, L.F. High-Intensity Respiratory Muscle Training Improves Strength and Dyspnea Poststroke: A Double-Blind Randomized Trial. Arch. Phys. Med. Rehabil. 2018, 100, 205-212. [CrossRef]

22. Cho, J.E.; Lee, H.J.; Kim, M.K.; Lee, W.H. The improvement in respiratory function by inspiratory muscle training is due to structural muscle changes in patients with stroke: A randomized controlled pilot trial. Top. Stroke Rehabil. 2017, 25, 37-43. [CrossRef]

23. Yoo, H.J.; Pyun, S.B. Efficacy of Bedside Respiratory Muscle Training in Patients with Stroke: A Randomized Controlled Trial. Am. J. Phys. Med. Rehabil. 2018, 97, 691-697. [CrossRef]

24. Lee, H.J.; Kang, T.W.; Kim, B.R. Effects of diaphragm and deep abdominal muscle exercise on walking and balance ability in patients with hemiplegia due to stroke. J. Exerc. Rehabil. 2018, 14, 648-653. [CrossRef]

25. Jung, K.M.; Bang, D.H. Effect of inspiratory muscle training on respiratory capacity and walking ability with subacute stroke patients: A randomized controlled pilot trial. J. Phys. Ther. Sci. 2017, 29, 336-339. [CrossRef] [PubMed] 
26. Jung, N.J.; Na, S.S.; Kim, S.K.; Hwangbo, G. The effect of the inspiratory muscle training on functional ability in stroke patients. J. Phys. Ther. Sci. 2017, 29, 1954-1956. [CrossRef] [PubMed]

27. Oh, D.; Kim, G.; Lee, W.; Shin, M.M.S. Effects of inspiratory muscle training on balance ability and abdominal muscle thickness in chronic stroke patients. J. Phys. Ther. Sci. 2016, 28, 107-111. [CrossRef] [PubMed]

28. Guillen-Sola, A.; Sartor, M.M.; Soler, N.B.; Duarte, E.; Barrera, M.C.; Marco, E. Respiratory muscle strength training and neuromuscular electrical stimulation in subacute dysphagic stroke patients: A randomized controlled trial. Clin. Rehabil. 2016, 31, 761-771. [CrossRef]

29. Chen, P.C.; Liaw, M.Y.; Wang, L.Y.; Tsai, Y.C.; Hsin, Y.J.; Chen, Y.C. Inspiratory muscle training in stroke patients with congestive heart failure A CONSORT-compliant prospective randomized single-blind controlled trial. Medicine 2016, 95, e4856. [CrossRef]

30. Messaggi-Sartor, M.; Guillen-Sola, A.; Depolo, M.; Duarte, E.; Rodríguez, D.A.; Barrera, M.-C.; Barreiro, E.; Escalada, F.; Orozco-Levi, M.; Marco, E. Inspiratory and expiratory muscle training in subacute stroke: A randomized clinical trial. Neurology 2015, 85, 564-572. [CrossRef]

31. Kulnik, S.T.; Birring, S.S.; Moxham, J.; Rafferty, G.F.; Kalra, L. Does Respiratory Muscle Training Improve Cough Flow in Acute Stroke? Pilot Randomized Controlled Trial. Stroke 2015, 46, 447-453. [CrossRef]

32. Kim, C.Y.; Lee, J.S.; Kim, H.D.; Kim, I.S. Effects of the combination of respiratory muscle training and abdominal drawing-in maneuver on respiratory muscle activity in patients with post-stroke hemiplegia: A pilot randomized controlled trial. Top. Stroke Rehabil. 2015, 22, 262-270. [CrossRef]

33. Yim, J.; Kim, J.; Park, J. Effects of Respiratory Muscle and Endurance Training Using an Individualized Training Device on the Pulmonary Function and Exercise Capacity in Stroke Patients. Med. Sci. Monit. 2014, 20, 2543-2549. [CrossRef] [PubMed]

34. Jung, J.-H.; Kim, N.-S. Effects of Inspiratory Muscle Training on Diaphragm Thickness, Pulmonary Function, and Chest Expansion in Chronic Stroke Patients. J. Korean Soc. Phys. Med. 2013, 8, 59-69. [CrossRef]

35. Britto, R.R.; Rezende, N.R.; Marinho, K.C.; Torres, J.L.; Parreira, V.F.; Teixeira-Salmela, L.F. Inspiratory Muscular Training in Chronic Stroke Survivors: A Randomized Controlled Trial. Arch. Phys. Med. Rehabil. 2011, 92, 184-190. [CrossRef] [PubMed]

36. Kim, K.; Fell, D.W.; Lee, J.H. Feedback Respiratory Training to Enhance Chest Expansion and Pulmonary Function in Chronic Stroke: A Double-Blind, Randomized Controlled Study. J. Phys. Ther. Sci. 2011, 23, 75-79. [CrossRef]

37. Sutbeyaz, S.T.; Koseoglu, F.; Inan, L.; Coskun, O. Respiratory muscle training improves cardiopulmonary function and exercise tolerance in subjects with subacute stroke: A randomized controlled trial. Clin. Rehabil. 2010, 24, 240-250. [CrossRef] [PubMed]

38. Sterne, J.A.; Sutton, A.J.; Ioannidis, J.P.A.; Terrin, N.; Jones, D.R.; Lau, J.; Carpenter, J.R.; Rücker, G.; Harbord, R.; Schmid, C.H.; et al. Recommendations for examining and interpreting funnel plot asymmetry in meta-analyses of randomised controlled trials. BMJ 2011, 343, d4002. [CrossRef] [PubMed]

39. Lanini, B.; Gigliotti, F.; Coli, C.; Bianchi, R.; Pizzi, A.; Romagnoli, I.; Grazzini, M.; Stendardi, L.; Scano, G. Dissociation between respiratory effort and dyspnoea in a subset of patients with stroke. Clin. Sci. 2002, 103, 467-473. [CrossRef] [PubMed]

40. Pollock, R.D.; Rafferty, G.F.; Moxham, J.; Kalra, L.; Rafferty, G.F. Respiratory Muscle Strength and Training in Stroke and Neurology: A Systematic Review. Int. J. Stroke 2012, 8, 124-130. [CrossRef]

41. De Almeida, I.C.L.; Clementino, A.C.C.R.; Rocha, E.H.T.; Brandão, D.C.; De Andrade, A.D. Effects of hemiplegy on pulmonary function and diaphragmatic dome displacement. Respir. Physiol. Neurobiol. 2011, 178, 196-201. [CrossRef]

42. Tomczak, C.R.; Jelani, A.; Haennel, R.G.; Haykowsky, M.; Welsh, R.; Manns, P. Cardiac Reserve and Pulmonary Gas Exchange Kinetics in Patients With Stroke. Stroke 2008, 39, 3102-3106. [CrossRef]

43. Schünemann, H.J.; Vist, G.E.; Higgins, J.P.; Santesso, N.; Deeks, J.J.; Glasziou, P.; Akl, E.A.; Guyatt, G.H. Interpreting results and drawing conclusions. In Cochrane Handbook for Systematic Reviews of Interventions; John Wiley \& Sons: Chichester, UK, 2019; pp. 403-431.

44. Reyes, A.; Ziman, M.; Nosaka, K.; Nosaka, K. Respiratory Muscle Training for Respiratory Deficits in Neurodegenerative Disorders. Chest 2013, 143, 1386-1394. [CrossRef]

45. Field, M.J.; Gebruers, N.; Shanmuga Sundaram, T.; Nicholson, S.; Mead, G. Physical Activity after Stroke: A Systematic Review and Meta-Analysis. ISRN Stroke 2013, 2013, 1-13. [CrossRef] 
46. Michaelsen, S.M.; Ovando, A.C.; Romaguera, F.; Ada, L. Effect of Backward Walking Treadmill Training on Walking Capacity after Stroke: A Randomized Clinical Trial. Int. J. Stroke 2014, 9, 529-532. [CrossRef] [PubMed]

47. Sulter, G.; Steen, C.; De Keyser, J. Use of the Barthel index and modified Rankin scale in acute stroke trials. Stroke 1999, 30, 1538-1541. [CrossRef] [PubMed]

48. Blum, L.; Korner-Bitensky, N. Usefulness of the Berg Balance Scale in Stroke Rehabilitation: A Systematic Review. Phys. Ther. 2008, 88, 559-566. [CrossRef]

(C) 2020 by the authors. Licensee MDPI, Basel, Switzerland. This article is an open access article distributed under the terms and conditions of the Creative Commons Attribution (CC BY) license (http://creativecommons.org/licenses/by/4.0/). 